\title{
n/p-harmonic maps Regularity for the sphere case
}

\section{Journal Article}

\section{Author(s):}

Da Lio, Francesca; Schikorra, Armin

Publication date:

2012-10

Permanent link:

https://doi.org/10.3929/ethz-b-000079415

\section{Rights / license:}

In Copyright - Non-Commercial Use Permitted

\section{Originally published in:}

Advances in Calculus of Variations 7(1), https://doi.org/10.1515/acv-2012-0107 


\title{
$n / p$-harmonic maps: Regularity for the sphere case
}

\author{
Francesca Da Lio and Armin Schikorra \\ Communicated by Giuseppe Mingione
}

Abstract. We introduce $n / p_{\alpha}$-harmonic maps as critical points of the energy

$$
\varepsilon_{n, p_{\alpha}}(v)=\int_{\mathbb{R}^{n}}\left|\Delta^{\frac{\alpha}{2}} v\right|^{p_{\alpha}}
$$

where pointwise $v: D \subset \mathbb{R}^{n} \rightarrow \mathbb{S}^{N-1}$, for the $N$-sphere $\mathbb{S}^{N-1} \subset \mathbb{R}^{N}$ and $p_{\alpha}=\frac{n}{\alpha}$. This energy combines the non-local behaviour of the fractional harmonic maps introduced by Rivière and the first author with the degenerate arguments of the $n$-Laplacian. In this setting, we will prove Hölder continuity.

Keywords. Harmonic maps, nonlinear elliptic PDE, regularity of solutions.

2010 Mathematics Subject Classification. 58E20, 35B65, 35J60, $35 \mathrm{~S} 05$.

\section{Introduction}

Our work is motivated by recent results $[3-5,16,18]$ which proved regularity for critical points of the energy $\mathcal{F}_{n}$ acting on maps $v: \mathbb{R}^{n} \rightarrow \mathbb{R}^{N}, n, N \in \mathbb{N}$, for closed manifolds $\mathcal{N} \subset \mathbb{R}^{N}$,

$$
\widetilde{F}_{n}(v)=\int_{\mathbb{R}^{n}}\left|\Delta^{\frac{n}{4}} v\right|^{2} \quad v \in \mathcal{N} \subset \mathbb{R}^{N} \text { a.e. }
$$

Here, the operator $\Delta^{\frac{\alpha}{2}} v$ is defined as a multiplier operator with symbol $-|\xi|^{\alpha}$, that is, denoting the Fourier transform and its inverse by $(\cdot)^{\wedge}$ and $(\cdot)^{\vee}$, respectively,

$$
\Delta^{\frac{\alpha}{2}} v=\left(-|\xi|^{\alpha} v^{\wedge}\right)^{\vee}
$$

These energies were introduced by $\mathrm{T}$. Rivière and the first author - and they can be seen as an $n$-dimensional alternative to the two-dimensional Dirichlet energy

$$
\mathscr{D}_{2}(v)=\int_{\mathbb{R}^{2}}|\nabla v|^{2}, \quad v \in \mathcal{N} \subset \mathbb{R}^{N} \text { a.e. }
$$

\footnotetext{
A. Schikorra has received funding from the European Research Council under the European Union's Seventh Framework Programme (FP7/2007-2013)/ERC grant agreement no 267087, DAAD PostDoc Program (D/10/50763) and the Forschungsinstitut für Mathematik, ETH Zürich.
} 
Both energies have critical Euler-Lagrange equations. That is, the highest order terms scale exactly as the lower-order terms, thus inhibiting the application of a general regularity theory based only on the general growth of the right-hand side - one has to consider the finer behaviour of the equation: these exhibit an antisymmetric structure, which is closely related to the appearance of Hardy spaces and compensated compactness - and induces regularity of critical points. In two dimensions, these facts were observed in Rivière's celebrated [14] for all conformally invariant variational functionals (of which the Dirichlet energy is a prototype). We refer the interested reader to the introductions of [4,5] for more on this.

Another possibility of generalizing the Dirichlet energy to arbitrary dimensions (whilst preserving the criticality of the Euler-Lagrange equations) is to consider

$$
\mathscr{D}_{n}(v)=\int_{\mathbb{R}^{n}}|\nabla v|^{n}, \quad v \in \mathcal{N} \subset \mathbb{R}^{N} \text { a.e. }
$$

Again in this case, the now degenerate Euler-Lagrange equations are critical and exhibit an antisymmetric structure, cf. [15, Chapter III] - only that it is not known so far, whether in general this structure implies even continuity. In fact, towards regularity of these systems, only few results are known. In [19] P. Strzelecki proved regularity if the target manifold is a round sphere $\mathbb{S}^{N-1}$ - which extended the respective Dirichlet-energy result by F. Hélein [10]. In the setting of general manifolds, we know so far of convergence results, cf. [21], and only under additional assumptions on the solution there are regularity results, cf. $[8,13,17]$.

It then seems interesting to consider an energy which combines the difficulties of $\mathscr{D}_{n}$ and $\mathscr{F}_{n}$. Namely we will work with

$$
\varepsilon_{n, p_{\alpha}}(v)=\int_{\mathbb{R}^{n}}\left|\Delta^{\frac{\alpha}{2}} v\right|^{p_{\alpha}},\left.\quad v\right|_{D} \in \mathcal{N} \subset \mathbb{R}^{N} \text { a.e., where } p_{\alpha}=\frac{n}{\alpha}, D \subset \subset \mathbb{R}^{n} .
$$

Note, in the Euler-Lagrange equations of $\mathcal{E}_{n, p_{\alpha}}$, the leading order differential operator is nonlocal and degenerate. Again, these setting are critical for regularity: one checks that any mapping $v$ with finite energy $\varepsilon_{n, p_{\alpha}}$ belongs to BMO, but does not necessarily need to be continuous, as Frehse's counterexample [9] shows.

Here we consider the situation of a sphere, i.e. $\mathcal{N}=\mathbb{S}^{N-1}$. Our main result is:

Theorem 1.1. Let $u$ be a critical point of $\varepsilon_{n, p_{\alpha}}$ as in (1.1), $p_{\alpha} \in(1, \infty)$. Then $u$ is Hölder-continuous.

For a special case of $p$-biharmonic maps into spheres there are earlier results by Strzelecki, see [20].

Naturally, one expects at least parts of this result to hold for more general manifolds $\mathcal{N}$. To this end, in [6] we will treat the case of general manifolds, but with the condition $p_{\alpha} \leq 2$. 
The proof relies on a suitable adaption of the arguments in $[3-5,16,18]$, the details of which we will explain in the next section: the Euler-Lagrange equations of a critical point, see [5,16], imply that

$$
\int_{\mathbb{R}^{n}}\left|\Delta^{\frac{\alpha}{2}} u\right|^{p_{\alpha}-2} \Delta^{\frac{\alpha}{2}} u^{i} \Delta^{\frac{\alpha}{2}}\left(\mu_{i j} u^{j} \varphi\right)=0
$$

for all $\varphi \in C_{0}^{\infty}(D), \mu_{i j}=-\mu_{j i} \in \mathbb{R}$. Note that the main difference and difficulty comparing this equation to the $n / 2$-harmonic case in $[5,16]$ is the weight

$$
\left|\Delta^{\frac{\alpha}{2}} u\right|^{p_{\alpha}-2} .
$$

Moreover, we have the sphere-condition,

$$
|u(x)|=1 \quad \text { for a.e. } x \in D .
$$

For a sketch of the proof, let us assume that $D=\mathbb{R}^{n}$. Note that (1.3) reveals information about the growth of the derivatives of $u$ in the direction of $u$ :

$$
u \cdot \nabla u \equiv 0 .
$$

Moreover - and more suitable to our case -

$$
-2 u \cdot \Delta^{\frac{\alpha}{2}} u=\left(\Delta^{\frac{\alpha}{2}}|u|^{2}-u \cdot \Delta^{\frac{\alpha}{2}} u-u \cdot \Delta^{\frac{\alpha}{2}} u\right)-\underbrace{\Delta^{\frac{\alpha}{2}} \overbrace{|u|^{2}}^{\equiv 1}}_{\equiv 0} .
$$

These 3-term commutators $H_{\alpha}(u, u)$ appearing on the right-hand side,

$$
H_{\alpha}(u, v):=\Delta^{\frac{\alpha}{2}}(u v)-u \Delta^{\frac{\alpha}{2}} v-v \Delta^{\frac{\alpha}{2}} u,
$$

have a special behaviour, which can best be seen by taking $\alpha=2: H_{2}(u, v)=$ $2 \nabla u \cdot \nabla v$ - they behave like products of lower-order operators applied to $u$ and $v$, for this interpretation cf. $[16,18]$. They are also closely related to the T1-Theorem and the "Leibniz rule" for fractional order derivatives obtained by Kato and Ponce, see [12] and [11, Corollary 1.2]. In our context, certain versions of these commutators were observed in [5] and estimated in Triebel- and Besov-spaces via fine estimates by paraproducts, cf. [6]. The necessary estimates for these operators can be paraphrased by

Theorem 1.2 (cf. [18]). For any $\alpha \in(0, n)$, let $u=\Delta^{-\frac{\alpha}{2}} \Delta^{\frac{\alpha}{2}} u, v=\Delta^{-\frac{\alpha}{2}} \Delta^{\frac{\alpha}{2}} v$. Then for $\alpha \in(0, n)$ there exists some constant $C_{\alpha}>0$ and a number $L \equiv L_{\alpha} \in \mathbb{N}$, 
and for $k \in\{1, \ldots, L\}$ there exist constants $s_{k} \in(0, \alpha), t_{k} \in\left[0, s_{k}\right]$ such that for any $i=1, \ldots, n$, where $\mathcal{R}_{i}$ denotes the Riesz transform,

$$
\left|\mathcal{R}_{i} H_{\alpha}(u, v)(x)\right| \leq C \sum_{k=1}^{L} M_{k} \Delta^{-\frac{s_{k}-t_{k}}{2}}\left(\Delta^{-\frac{t_{k}}{2}}\left|\Delta^{\frac{\alpha}{2}} u\right| N_{k} \Delta^{-\frac{\alpha}{2}+\frac{s_{k}}{2}}\left|\Delta^{\frac{\alpha}{2}} v\right|\right) .
$$

Here, $M_{k}, N_{k}$ are possibly Riesz transforms, or the identity. Moreover, $\left|s_{k}-t_{k}\right|$ can be supposed to be arbitrarily small. In particular, for any $\alpha \in(0, n)$ and any $q, q_{1}, q_{2} \in[1, \infty]$ such that

$$
\frac{1}{q}=\frac{1}{q_{1}}+\frac{1}{q_{2}}
$$

we have

$$
\left\|H_{\alpha}(u, v)\right\|_{\left(\frac{n}{\alpha}, q\right), \mathbb{R}^{n}} \prec\left\|\Delta^{\frac{\alpha}{2}} u\right\|_{\left(\frac{n}{\alpha}, q_{2}\right), \mathbb{R}^{n}}\left\|\Delta^{\frac{\alpha}{2}} v\right\|_{\left(\frac{n}{\alpha}, q_{2}\right), \mathbb{R}^{n}} .
$$

Here, $\|\cdot\|_{(p, q)}$ denotes the Lorentz-space $L^{p, q}\left(\mathbb{R}^{n}\right)$-norm.

Consequently, (1.3) controls $u \cdot \Delta^{\frac{\alpha}{2}} u$ roughly like

$$
\left\|u \cdot \Delta^{\frac{\alpha}{2}} u\right\|_{p_{\alpha}, \mathbb{R}^{n}} \prec\left\|\Delta^{\frac{\alpha}{2}} u\right\|_{p_{\alpha}, \mathbb{R}^{n}}^{2} .
$$

This argument can be localized and then implies an estimate for the growth $u \cdot \Delta^{\frac{\alpha}{2}} u$ in the $L^{p_{\alpha}}$-norm on small balls by the square $\left\|\Delta^{\frac{\alpha}{2}} u\right\|_{p_{\alpha}}^{2}$ localized essentially to slightly bigger balls.

Now the fact that $|u| \equiv 1$ implies also that in order to control the growth of $\Delta^{\frac{\alpha}{2}} u$, it suffices to estimate the growth of $u \cdot \Delta^{\frac{\alpha}{2}} u$ and the growth of $\mu_{i j} u^{i} \Delta^{\frac{\alpha}{2}} u^{j}$ for finitely many $\mu_{i j}=-\mu_{j i} \in \mathbb{R}$, see Proposition B.1. But terms of the form $\mu_{i j} u^{i} \Delta^{\frac{\alpha}{2}} u^{j}$ can be estimated by the Euler-Lagrange equation (1.2).

By this kind of argument, we obtain (essentially) the following growth estimates for all balls $B_{r}$ :

$$
\begin{aligned}
\left\|\Delta^{\frac{\alpha}{2}} u\right\|_{p_{\alpha}, B_{r}} \leq\left\|\Delta^{\frac{\alpha}{2}} u\right\|_{p_{\alpha}, B_{\Lambda r}}^{2} & \\
& +\Lambda^{-\gamma}\left\|\Delta^{\frac{\alpha}{2}} u\right\|_{p_{\alpha}, \mathbb{R}^{n}} \sum_{k=1}^{\infty} 2^{-\gamma k}\left\|\Delta^{\frac{\alpha}{2}} u\right\|_{p_{\alpha}, B_{2^{k} \Lambda} \backslash B_{2^{k}} \Lambda r},
\end{aligned}
$$

for some $\gamma>0$, and any $\Lambda>2$. Using an iteration technique, this implies that

$$
\left\|\Delta^{\frac{\alpha}{2}} u\right\|_{p_{\alpha}, B_{r}} \leq C r^{\alpha},
$$

which accounts for the Hölder-continuity of $u$. 
Let us briefly underline the differences with respect to the manifold case treated in [6]. There, instead of using such a simple condition (1.3), we are forced to work with more general projections in the tangential and normal components of $\Delta^{\frac{\alpha}{2}} u$ (that is, we work with projections related to the derivatives of $u$ ). This seems to prohibit extremely small $\alpha$ (which in the sphere case pose no problem). On the other hand, the respective Euler-Lagrange equations actually exhibit a non-trivial right-hand side with antisymmetric structure. This will force us to estimate the growth of $\Delta^{\frac{\alpha}{2}} u$ in the weak (Lorentz) space $L^{p_{\alpha}, \infty}$, which in turn will make it necessary to gain $L^{p_{\alpha}, 1}$-estimates from the three-term commutators $H_{\alpha}(\cdot, \cdot)$. This again, cf. (1.4) for $q_{1}=q_{2}=2$, will only be possible if $p_{\alpha} \leq 2$.

We will use notation similar to [16]: We say that $A \subset \subset \mathbb{R}^{n}$ if $A$ is a bounded subset of $\mathbb{R}^{n}$. For a set $A \subset \mathbb{R}^{n}$ we will denote its $n$-dimensional Lebesgue measure by $|A|$. By $B_{r}(x) \subset \mathbb{R}^{n}$ we denote the open ball with radius $r$ and center $x \in \mathbb{R}^{n}$. If no confusion arises, we will abbreviate $B_{r} \equiv B_{r}(x)$. If $p \in[1, \infty]$, we usually will denote by $p^{\prime}$ the Hölder conjugate, that is, $\frac{1}{p}+\frac{1}{p^{\prime}}=1$. By $f * g$ we denote the convolution of two functions $f$ and $g$. Lastly, our constants - frequently denoted by $C$ or $c$ - can possibly change from line to line and usually depend on the space dimensions involved, further dependencies will be denoted by a subscript, though we will make no effort to pin down the exact value of those constants. If we consider the constant factors to be irrelevant with respect to the mathematical argument, for the sake of simplicity we will omit them in the calculations, writing $\prec, \succ, \approx$ instead of $\leq, \geq$ and $=$.

We will use the same cutoff-functions as in, e.g., $[5,16]: \eta_{r}^{k} \in C_{0}^{\infty}\left(A_{r, k}\right)$ where

$$
B_{r, k}(x):=B_{2^{k} r}(x)
$$

for $k \geq 1$,

$$
A_{r, k}(x):=B_{r, k+1}(x) \backslash B_{r, k-1}(x),
$$

and for $k=0$

$$
A_{r, 0}(x):=B_{r, 0}(x) .
$$

Moreover, $\sum_{k} \eta_{r}^{k} \equiv 1$ pointwise everywhere, and we assume that

$$
\left|\nabla^{l} \eta_{r}^{k}\right| \leq C_{l}\left(2^{k} r\right)^{-l}, \quad \text { for } l \in \mathbb{N} .
$$

\section{Proof of Theorem 1.1}

Let $\alpha \in(0, n), p_{\alpha}=\frac{n}{\alpha} \in(1, \infty)$, and $u \in L^{p_{\alpha}}\left(\mathbb{R}^{n}, \mathbb{R}^{N}\right), \Delta^{\frac{\alpha}{2}} u \in L^{p_{\alpha}}\left(\mathbb{R}^{n}, \mathbb{R}^{N}\right)$. Assume moreover that $D \subset \subset \mathbb{R}^{n}$ such that (1.2), (1.3) hold.

As (1.2) and (1.3) are equations satisfied by any critical point $u$ of Theorem 1.1, we have to show the following. 
Theorem 2.1. Let $u \in L^{p_{\alpha}}\left(\mathbb{R}^{n}, \mathbb{R}^{N}\right), \Delta^{\frac{\alpha}{2}} u \in L^{p}\left(\mathbb{R}^{n}, \mathbb{R}^{N}\right)$ satisfy (1.2), (1.3). Then $u$ is Hölder continuous in $D$.

In order to prove Theorem 2.1, we first rewrite equations (1.2) and (1.3) in a fashion similar to $[5,16]$ : Firstly, equation (1.2) is equivalent to

$$
\int_{\mathbb{R}^{n}}\left|\Delta^{\frac{\alpha}{2}} u\right|^{p_{\alpha}-2} u^{j} \mu_{i j} \Delta^{\frac{\alpha}{2}} u^{i} \Delta^{\frac{\alpha}{2}} \varphi=-\int_{\mathbb{R}^{n}}\left|\Delta^{\frac{\alpha}{2}} u\right|^{p_{\alpha}-2} \Delta^{\frac{\alpha}{2}} u^{i} \mu_{i j} H\left(u^{j}, \varphi\right),
$$

for all $\varphi \in C_{0}^{\infty}(D), \mu_{i j}=-\mu_{j i} \in \mathbb{R}$. Here and henceforth,

$$
H(a, b) \equiv H_{\alpha}(a, b) \equiv \Delta^{\frac{\alpha}{2}}(a b)-a \Delta^{\frac{\alpha}{2}} b-b \Delta^{\frac{\alpha}{2}} a .
$$

Assume we proven Hölder-continuity of $u$ in a ball $B \subset \subset D$. Pick a slightly bigger ball $\tilde{B} \subset \subset D, \tilde{B} \supset \supset B$, and let $w:=\bar{\eta} u$, for some

$$
\bar{\eta} \in C_{0}^{\infty}(D,[0,1]), \quad \bar{\eta} \equiv 1 \text { on } \tilde{B} .
$$

Note that $w \in L^{p}\left(\mathbb{R}^{n}\right)$ for any $p \in[1, \infty]$. It suffices to show Hölder regularity for $w$. The relevant equations for $w$ stemming from (1.3) and (2.1) are then (again, cf. $[5,16])$

$$
w \cdot \Delta^{\frac{\alpha}{2}} w=\frac{1}{2} H(w, w)+\frac{1}{2} \Delta^{\frac{\alpha}{2}} \bar{\eta}^{2} \quad \text { a.e. in } \mathbb{R}^{n},
$$

and for all $\varphi \in C_{0}^{\infty}(D), \mu_{i j}=-\mu_{j i} \in \mathbb{R}$,

$$
\begin{aligned}
& \int_{\mathbb{R}^{n}}\left|\Delta^{\frac{\alpha}{2}} w\right|^{p_{\alpha}-2} w^{j} \mu_{i j} \Delta^{\frac{\alpha}{2}} w^{i} \Delta^{\frac{\alpha}{2}} \varphi \\
& =\mu_{i j} \int_{\mathbb{R}^{n}}\left(\left|\Delta^{\frac{\alpha}{2}} w\right|^{p_{\alpha}-2} \Delta^{\frac{\alpha}{2}} w^{i}-\left|\Delta^{\frac{\alpha}{2}} u\right|^{p_{\alpha}-2} \Delta^{\frac{\alpha}{2}} u^{i}\right) w^{j} \Delta^{\frac{\alpha}{2}} \varphi \\
& \quad+\mu_{i j} \int_{\mathbb{R}^{n}}\left(\left|\Delta^{\frac{\alpha}{2}} u\right|^{p_{\alpha}-2} \Delta^{\frac{\alpha}{2}} u^{i}\right)\left(w^{j}-u^{j}\right) \Delta^{\frac{\alpha}{2}} \varphi \\
& \quad+\mu_{i j} \int_{\mathbb{R}^{n}}\left|\Delta^{\frac{\alpha}{2}} u\right|^{p_{\alpha}-2} \Delta^{\frac{\alpha}{2}} u^{i} H\left(w^{j}-u^{j}, \varphi\right) \\
& \quad+\mu_{i j} \int_{\mathbb{R}^{n}}\left(\left|\Delta^{\frac{\alpha}{2}} w\right|^{p_{\alpha}-2} \Delta^{\frac{\alpha}{2}} w^{i}-\left|\Delta^{\frac{\alpha}{2}} u\right|^{p_{\alpha}-2} \Delta^{\frac{\alpha}{2}} u^{i}\right) H\left(w^{j}, \varphi\right) \\
& \quad-\mu_{i j} \int_{\mathbb{R}^{n}}\left|\Delta^{\frac{\alpha}{2}} w\right|^{p_{\alpha}-2} \Delta^{\frac{\alpha}{2}} w^{i} H\left(w^{j}, \varphi\right) .
\end{aligned}
$$

Now we need to appropriately adapt several arguments of $[5,16]$ : first of all, using (2.2) we control $\Delta^{\frac{\alpha}{2}} w$ projected into the orthogonal space to the sphere at the point $w, T_{w}^{\perp} \mathbb{S}^{N-1}$. 


\section{The orthogonal part}

Namely, from (2.2) and Lemma A.9 one infers

Lemma 2.2. There is $\gamma=\gamma_{\alpha . p_{\alpha}}>0$ and a constant $C$ depending on the choice of $B, \tilde{B}, \bar{\eta}$, such that the following holds: for any $\varepsilon>0$ there exist $\Lambda>0, R>0$, such that for any $B_{\Lambda} \subset \subset \subset B, r \in(0, R)$,

$$
\begin{aligned}
\left\|w \cdot \Delta^{\frac{\alpha}{2}} w\right\|_{p_{\alpha}, B_{r}} \leq \varepsilon\left\|\Delta^{\frac{\alpha}{2}} w\right\|_{p_{\alpha}, B_{\Lambda r}}+C r^{\gamma} & \\
& +\varepsilon \sum_{k=1}^{\infty} 2^{-\gamma k}\left\|\Delta^{\frac{\alpha}{2}} w\right\|_{p_{\alpha}, B_{2^{k} \Lambda r} \backslash B_{2^{k-1} \Lambda r}} .
\end{aligned}
$$

The next step is to control the tangential part of $\Delta^{\frac{\alpha}{2}} w$ by means of (2.3). The terms on the right-hand side of (2.3) can be divided into two groups. The integrands of (2.4), (2.5), (2.6), and (2.7) always contain differences of the form $w-u$ which is trivial in $\tilde{B}$. Consequently, we show that these terms behave subcritical.

\section{Estimates of tangential part: Subcritical terms (2.4), (2.5), (2.6), (2.7)}

In order to be more precise, we need the following proposition, which can be proven by an method which appears in a similar form already in [5].

Proposition 2.3 (Estimates for disjoint-support terms). Let $r \in(0,1), d>0$ and $p, q \in[1, \infty)$ such that $B_{r+d} \subset \tilde{B}$. Then, for any $f, \Delta^{\frac{\alpha}{2}} f \in L^{q}\left(\mathbb{R}^{n}\right)$,

$$
\left\|\Delta^{\frac{\alpha}{2}}((1-\bar{\eta}) f)\right\|_{p, B_{r}} \prec C_{d} r^{\frac{n}{p}}\left(\|f\|_{q, \mathbb{R}^{n}}+\left\|\Delta^{\frac{\alpha}{2}} f\right\|_{q, \mathbb{R}^{n}}\right) .
$$

If moreover supp $f \subset B_{r}$, for some $\gamma=\gamma_{\alpha}$,

$$
\left\|(1-\bar{\eta}) \Delta^{\frac{\alpha}{2}} f\right\|_{\infty, \mathbb{R}^{n}} \prec C_{d} r^{\gamma}\left\|\Delta^{\frac{\alpha}{2}} f\right\|_{q} .
$$

Proof. Instead of using Fourier-transform as in $[5,16]$ etc., we use the following strategy: let $\alpha:=2 K+s$, where $s \in(0,2)$ (the case $s=0$ is trivial), $K \in \mathbb{N}$, that is,

$$
\Delta^{\frac{\alpha}{2}}=\Delta^{\frac{s}{2}} \Delta^{K} .
$$

Set $h:=\Delta^{K} g$, and recall that (cf., e.g., [7, Lemma 3.2])

$$
\Delta^{\frac{s}{2}} h(x)=c \int \frac{h(x+z)+h(x-z)-2 h(x)}{|z|^{n+s}} d z .
$$

Thus, if supp $g \subset \mathbb{R}^{n} \backslash B_{r+d}$ and $x \in B_{r}$, as is the case in (2.9), or supp $g \subset B_{r}$ and $x \in \mathbb{R}^{n} \backslash B_{r+d}$, as is the case in (2.10)

$$
\left|\Delta^{\frac{s}{2}} h(x)\right| \prec \int_{|z|>d}|h(x+z)||z|^{-n-s} d z=|h| *\left(|\cdot|^{-n-s} \chi|\cdot|>d\right)(x),
$$


and

$$
\begin{aligned}
\left\||h| *\left(|\cdot|^{-n-s} \chi|\cdot|>d\right)\right\|_{p} & \prec\|h\|_{q} d^{-n\left(q_{2}-1\right)-2 s q_{2}} \\
& \prec d^{-n\left(q_{2}-1\right)-2 s q_{2}}\left(\|f\|_{q, \mathbb{R}^{n}}+\left\|\Delta^{K} f\right\|_{q, \mathbb{R}^{n}}\right),
\end{aligned}
$$

where $q_{2}$ is chosen such that

$$
1+\frac{1}{p}=\frac{1}{q}+\frac{1}{q_{2}}
$$

For (2.9) we then use the fact that $W^{\alpha, q}=W^{2 K+s, q} \subset W^{2 K, q}$, see for example [7, Proposition 2.1], that is

$$
\|f\|_{q, \mathbb{R}^{n}}+\left\|\Delta^{K} f\right\|_{q, \mathbb{R}^{n}} \prec\|f\|_{q, \mathbb{R}^{n}}+\left\|\Delta^{\frac{\alpha}{2}} f\right\|_{q, \mathbb{R}^{n}} .
$$

For (2.9), note that by Poincaré's inequality,

$$
\|f\|_{q, \mathbb{R}^{n}}+\left\|\Delta^{K} f\right\|_{q, \mathbb{R}^{n}} \prec\left\|\Delta^{K} f\right\|_{q, \mathbb{R}^{n}} \prec r^{\alpha-K}\left\|\Delta^{\frac{\alpha}{2}} f\right\|_{q, \mathbb{R}^{n}} .
$$

Now, assume $\left|\mu_{i j}\right| \leq 2$. We claim that for any $\varphi \in C_{0}^{\infty}\left(B_{r}\right)$, where $B_{2 r} \subset B$, all but the last term on the right-hand side of (2.3) can be estimated by $r^{\gamma}$ for some $\gamma$, up to a constant factor depending on $u, B$, and the distance between $B_{r}$ and $\partial \tilde{B}$ :

Proposition 2.4 (Subcritical terms). There exists a constant $C$ depending on $u, B$, $\tilde{B}$, the choice of $\bar{\eta}$, and an exponent $\gamma \equiv \gamma_{p_{\alpha}, \alpha}>0$ such that for any $\varphi \in C_{0}^{\infty}\left(B_{r}\right)$, $\left\|\Delta^{\frac{\alpha}{2}} \varphi\right\|_{p_{\alpha}} \leq 1$ for arbitrary $B_{2 r} \subset B$, if

$$
\begin{aligned}
I & :=\int_{\mathbb{R}^{n}}\left(\left|\Delta^{\frac{\alpha}{2}} w\right|^{p_{\alpha}-2} \Delta^{\frac{\alpha}{2}} w^{i}-\left|\Delta^{\frac{\alpha}{2}} u\right|^{p_{\alpha}-2} \Delta^{\frac{\alpha}{2}} u^{i}\right) w^{j} \Delta^{\frac{\alpha}{2}} \varphi, \\
I I & :=\int_{\mathbb{R}^{n}}\left(\left|\Delta^{\frac{\alpha}{2}} u\right|^{p_{\alpha}-2} \Delta^{\frac{\alpha}{2}} u^{i}\right)\left(w^{j}-u^{j}\right) \Delta^{\frac{\alpha}{2}} \varphi, \\
I I I & :=\int_{\mathbb{R}^{n}}\left|\Delta^{\frac{\alpha}{2}} u\right|^{p_{\alpha}-2} \Delta^{\frac{\alpha}{2}} u^{i} H\left(w^{j}-u^{j}, \varphi\right), \\
I V & :=\int_{\mathbb{R}^{n}}\left(\left|\Delta^{\frac{\alpha}{2}} w\right|^{p_{\alpha}-2} \Delta^{\frac{\alpha}{2}} w^{i}-\left|\Delta^{\frac{\alpha}{2}} u\right|^{p_{\alpha}-2} \Delta^{\frac{\alpha}{2}} u^{i}\right) H\left(w^{j}, \varphi\right),
\end{aligned}
$$

then

$$
|I|+|I I|+|I I I|+|I V| \leq C r^{\gamma} .
$$

Proof. Note that for any $a, b \in \mathbb{R}^{N}$ we have

$$
\left.|| a\right|^{p-2} a-|b|^{p-2} b \mid \leq C_{p} \begin{cases}|a-b|^{p-1} & \text { if } p \in[1,2] \\ |a-b|^{p-1}+|a-b||b|^{p-2} & \text { if } p>2\end{cases}
$$


Now we argue via Proposition 2.3, using that there exists a constant $d=d_{B, \tilde{B}}>0$ such that $\operatorname{dist}\left(\operatorname{supp} \varphi, \mathbb{R}^{n} \backslash \tilde{B}\right)>d$. This is straightforward for $I$ and $I V$ (recall that $w \in L^{\infty}$ for $I$ and use Proposition A.7 for $I V$ ). For $I I I$, we apply Proposition 2.3 to the terms

$$
H\left(w^{j}-u^{j}, \varphi\right)=H\left((\bar{\eta}-1) u^{j}, \varphi\right)=\varphi \Delta^{\frac{\alpha}{2}}\left((\bar{\eta}-1) u^{j}\right)+(\bar{\eta}-1) u^{j} \Delta^{\frac{\alpha}{2}} \varphi,
$$

and finally, for $I I$ we first estimate

$$
\left\|(1-\bar{\eta}) u \Delta^{\frac{\alpha}{2}} \varphi\right\|_{p_{\alpha}} \prec\|u\|_{p_{\alpha}}\left\|(1-\bar{\eta}) \Delta^{\frac{\alpha}{2}} \varphi\right\|_{\infty} .
$$

\section{Estimates of tangential part - the critical term (2.8)}

In order to estimate the tangential part of $\Delta^{\frac{\alpha}{2}} w$ completely, we now need to control the last term (2.8), which is done in the following two propositions.

Proposition 2.5. There is a constant $C_{\alpha}, R>0$ and an exponent $\gamma>0$ such that the following holds. Let $\|w\|_{\infty} \leq 1$, and assume $\Delta^{\frac{\alpha}{2}} w \in L^{p_{\alpha}}\left(\mathbb{R}^{n}\right)$. Then for any $\varphi \in C_{0}^{\infty}\left(B_{r}\right), \Lambda \geq 5, r \in(0, R)$

$$
\|H(w, \varphi)\|_{p_{\alpha}, B_{2^{k} \Lambda r} \backslash B_{2^{k-1} \Lambda r}} \leq C\left(2^{k} \Lambda\right)^{-\gamma}\left\|\Delta^{\frac{\alpha}{2}} \varphi\right\|_{p_{\alpha}}
$$

Proof. We have on $B_{2^{k}} \Lambda r \backslash B_{2^{k-1}} \Lambda r$

$$
H(w, \varphi)=\Delta^{\frac{\alpha}{2}}(w \varphi)-w \Delta^{\frac{\alpha}{2}} \varphi .
$$

One checks that (exploiting the disjoint support via similar arguments as in Proposition 2.3)

$$
\left\|\Delta^{\frac{\alpha}{2}}(w \varphi)\right\|_{p_{\alpha}, B_{2} \Lambda_{\Lambda}} \backslash B_{2^{k-1} \Lambda r} \prec\|w\|_{\infty}\left(2^{k} \Lambda\right)^{-n}
$$

and as well

$$
\left\|w \Delta^{\frac{\alpha}{2}} \varphi\right\|_{p_{\alpha}, B_{2^{k} \Lambda r} \backslash B_{2^{k-1} \Lambda r}} \prec\|w\|_{\infty}\left(2^{k} \Lambda\right)^{-n}
$$

Moreover:

Proposition 2.6. There is a constant $C_{\alpha}$ and an exponent $\gamma>0$ such that the following holds. Let $\|w\|_{\infty} \leq 1$, and assume that $\Delta^{\frac{\alpha}{2}} w \in L^{p_{\alpha}}\left(\mathbb{R}^{n}\right)$. Then for any $\varphi \in C_{0}^{\infty}\left(B_{r}\right), \Lambda \geq 5$

$$
\|H(w, \varphi)\|_{p_{\alpha}, B_{\Lambda} r} \leq C\left(\Lambda^{-\gamma}\left\|\Delta^{\frac{\alpha}{2}} w\right\|_{p_{\alpha}}+\left\|\eta_{\Lambda^{3} r} \Delta^{\frac{\alpha}{2}} w\right\|_{p_{\alpha}}\right)\left\|\Delta^{\frac{\alpha}{2}} \varphi\right\|_{p_{\alpha}} .
$$

Proof. Set

$$
w=\Delta^{-\frac{\alpha}{2}}\left(\eta_{\Lambda^{3} r} \Delta^{\frac{\alpha}{2}} w\right)+\Delta^{-\frac{\alpha}{2}}\left(\left(1-\eta_{\Lambda^{3} r}\right) \Delta^{\frac{\alpha}{2}} w\right)=: w_{1}+w_{2},
$$


and

$$
\varphi=\Delta^{-\frac{\alpha}{2}}\left(\eta_{\Lambda r} \Delta^{\frac{\alpha}{2}} \varphi\right)+\Delta^{-\frac{\alpha}{2}}\left(\left(1-\eta_{\Lambda r}\right) \Delta^{\frac{\alpha}{2}} \varphi\right)=: \varphi_{1}+\varphi_{2} .
$$

Then

$$
H(w, \varphi)=H\left(w_{1}, \varphi\right)+H\left(w_{2}, \varphi_{2}\right)+H\left(w_{2}, \varphi_{1}\right) .
$$

We compute via Proposition A.7,

$$
\begin{aligned}
\left\|H\left(w_{1}, \varphi\right)\right\|_{p_{\alpha}} & \prec\left\|\eta_{\Lambda^{3} r} \Delta^{\frac{\alpha}{2}} w\right\|_{p_{\alpha}}\left\|\Delta^{\frac{\alpha}{2}} \varphi\right\|_{p_{\alpha}}, \\
\left\|H\left(w_{2}, \varphi_{2}\right)\right\|_{p_{\alpha}} & \prec\left\|\Delta^{\frac{\alpha}{2}} w\right\|_{p_{\alpha}}\left\|\left(1-\eta_{\Lambda r}\right) \Delta^{\frac{\alpha}{2}} \varphi\right\|_{p_{\alpha}} \\
& \prec \Lambda^{-n}\left\|\Delta^{\frac{\alpha}{2}} w\right\|_{p_{\alpha}}\left\|\Delta^{\frac{\alpha}{2}} \varphi\right\|_{p_{\alpha}} .
\end{aligned}
$$

In order to estimate $\left\|H\left(w_{2}, \varphi_{1}\right)\right\|_{p_{\alpha}}$ in the given set, according to Lemma A.6, similar to the arguments in [18], it suffices to estimate terms of the following form, for some $\psi \in C_{0}^{\infty}\left(B_{r}\right),\|\psi\|_{p_{\alpha^{\prime}}} \leq 1$, and for $s \in(0, \alpha), t \in[0, s]$ (in fact, there might appear additional 0-multipliers, but as they do not interfere with the argument, we ignore this for the sake of readability)

$$
\begin{aligned}
& \int \Delta^{-\frac{s-t}{2}} \psi \Delta^{-\frac{s}{2}}\left|\left(1-\eta_{\Lambda^{3} r}\right) \Delta^{\frac{\alpha}{2}} w\right| \Delta^{-\frac{\alpha-t}{2}}\left|\eta_{\Lambda r} \Delta^{\frac{\alpha}{2}} \varphi\right| \\
& =\int \Delta^{-\frac{s-t}{2}} \psi \eta_{\Lambda^{2} r} \Delta^{-\frac{t}{2}}\left|\left(1-\eta_{\Lambda^{3} r}\right) \Delta^{\frac{\alpha}{2}} w\right| \Delta^{-\frac{\alpha-s}{2}}\left|\eta_{\Lambda r} \Delta^{\frac{\alpha}{2}} \varphi\right| \\
& \quad+\int\left(1-\eta_{\Lambda^{2} r}\right) \Delta^{-\frac{s-t}{2}} \psi \Delta^{-\frac{t}{2}}\left|\left(1-\eta_{\Lambda^{3} r}\right) \Delta^{\frac{\alpha}{2}} w\right| \Delta^{-\frac{\alpha-s}{2}}\left|\eta_{\Lambda r} \Delta^{\frac{\alpha}{2}} \varphi\right| \\
& \prec\left\|\eta_{\Lambda^{2} r} \Delta^{-\frac{t}{2}}\left|\left(1-\eta_{\Lambda^{3} r}\right) \Delta^{\frac{\alpha}{2}} w\right|\right\|_{\frac{n}{\alpha-t}}\left\|\Delta^{\frac{\alpha}{2}} \varphi\right\|_{p_{\alpha}} \\
& \quad+\left\|\left(1-\eta_{\Lambda^{2} r}\right) \Delta^{-\frac{s-t}{2}} \psi\right\|_{\frac{n}{n-s+t-\alpha}}\left\|\Delta^{\frac{\alpha}{2}} w\right\|_{p_{\alpha}}\left\|\Delta^{\frac{\alpha}{2}} \varphi\right\|_{p_{\alpha}} \\
& \prec\left(\Lambda^{t-\alpha}+\Lambda^{-\alpha}\right)\left\|\Delta^{\frac{\alpha}{2}} w\right\|_{p_{\alpha}}\left\|\Delta^{\frac{\alpha}{2}} \varphi\right\|_{p_{\alpha}} .
\end{aligned}
$$

Here, we have used several times the techniques for products of (non-local) fractional operators with disjoint support, for the details of which we refer to, e.g., the arguments of Proposition 2.3 or [18, Proposition 4.4].

Setting $\gamma:=\min \{\alpha-t, \alpha, n\}$, we conclude.

\section{Conclusion for the tangential part}

By Proposition 2.4, Proposition 2.5 and Proposition 2.6, we arrive at the following

Proposition 2.7 (Estimate of (2.8)). There is $\gamma=\gamma_{\alpha \cdot p_{\alpha}}>0$ and a constant $C$ depending on the choice of $B, \tilde{B}, \bar{\eta}$, such that the following holds: for any $\varepsilon>0$ 
there exists $\Lambda>0, R>0$, such that for any $B_{r} \subset \subset B, r \in(0, R), \varphi \in C_{0}^{\infty}\left(B_{r}\right)$, $\left\|\Delta^{\frac{\alpha}{2}} \varphi\right\|_{p_{\alpha}} \leq 1, \mu_{i j}=-\mu j i,|\mu| \leq 1$,

$$
\begin{aligned}
& \mu_{i j} \int_{\mathbb{R}^{n}}\left|\Delta^{\frac{\alpha}{2}} w\right|^{p_{\alpha}-2} w^{j} \Delta^{\frac{\alpha}{2}} w^{i} \Delta^{\frac{\alpha}{2}} \varphi \\
& \quad \leq \varepsilon\left\|\Delta^{\frac{\alpha}{2}} w\right\|_{p_{\alpha}, B_{\Lambda} r}^{p_{\alpha}-1}+C r^{\gamma}+\varepsilon \sum_{k=1}^{\infty} 2^{-\gamma k}\left\|\Delta^{\frac{\alpha}{2}} w\right\|_{p_{\alpha}, B_{2}{ }^{k} \Lambda r}^{p_{\alpha}-1} \backslash B_{2^{k-1} \Lambda r}
\end{aligned}
$$

Now we can proceed by virtually the same arguments as in $[5,16]$ : firstly, Proposition 2.7 finally implies

Lemma 2.8. There is $\gamma=\gamma_{\alpha \cdot p_{\alpha}}>0$ and a constant $C$ depending on the choice of $B, \tilde{B}, \bar{\eta}$, such that the following holds: for any $\varepsilon>0$ there exists $\Lambda>0, R>0$, such that for any $B_{r} \subset \subset B, r \in(0, R), \mu_{i j}=-\mu j i,|\mu| \leq 1$,

$$
\begin{aligned}
& \left\|\left|\Delta^{\frac{\alpha}{2}} w\right|^{p_{\alpha}-2} \mu_{i j} w^{j} \Delta^{\frac{\alpha}{2}} w^{i}\right\|_{p_{\alpha^{\prime}}, B_{\Lambda^{-1} r}} \\
& \leq \varepsilon\left\|\Delta^{\frac{\alpha}{2}} w\right\|_{p_{\alpha}, B_{\Lambda} r}^{p_{\alpha}-1}+C r^{\gamma}+\varepsilon \sum_{k=1}^{\infty} 2^{-\gamma k}\left\|\Delta^{\frac{\alpha}{2}} w\right\|_{p_{\alpha}, B_{2^{k} \Lambda r} \backslash B_{2^{k-1} \Lambda r}}^{p_{\alpha}-1} .
\end{aligned}
$$

\section{Putting tangential and normal part together}

Together, Lemma 2.8 and Lemma 2.2 imply

Lemma 2.9. There is $\gamma=\gamma_{\alpha . p_{\alpha}}>0$ and a constant $C$ depending on the choice of $B, \tilde{B}, \bar{\eta}$, such that the following holds: for any $\varepsilon>0$ there exists $\Lambda>0, R>0$, such that for any $B_{r} \subset \subset B, r \in(0, R)$,

$$
\begin{aligned}
\left\|\Delta^{\frac{\alpha}{2}} w^{i}\right\|_{p_{\alpha}, B_{\Lambda^{-1} r}}^{p_{\alpha}-1} \leq \varepsilon\left\|\Delta^{\frac{\alpha}{2}} w\right\|_{p_{\alpha}, B_{\Lambda r}}^{p_{\alpha}-1}+C r^{\gamma} & \\
& +\varepsilon \sum_{k=1}^{\infty} 2^{-\gamma k}\left\|\Delta^{\frac{\alpha}{2}} w\right\|_{p_{\alpha}, B_{2^{k} \Lambda r} \backslash B_{2^{k-1} \Lambda r}}^{p_{\alpha}-1} .
\end{aligned}
$$

Proof. Note that, if $p_{\alpha} \geq 2$,

$$
\begin{aligned}
\left\|\mu_{i j} w^{j} \Delta^{\frac{\alpha}{2}} w^{i}\right\|_{p_{\alpha}, B_{\Lambda^{-1} r}}^{p_{\alpha}-1} & =\left\|\left|\mu_{i j} w^{j} \Delta^{\frac{\alpha}{2}} w^{i}\right|^{p_{\alpha}-2} \mu_{i j} w^{j} \Delta^{\frac{\alpha}{2}} w^{i}\right\|_{p_{\alpha^{\prime}, B_{\Lambda^{-1} r}}} \\
& \prec\left\|\left|\Delta^{\frac{\alpha}{2}} w\right|^{p_{\alpha}-2} \mu_{i j} w^{j} \Delta^{\frac{\alpha}{2}} w^{i}\right\|_{p_{\alpha^{\prime}, B_{\Lambda}{ }^{-1} r}} .
\end{aligned}
$$

On the other hand, if $p_{\alpha} \in(1,2)$, we set for $\theta>0$ from Proposition B.1,

$$
A_{\mu, \theta}:=\left\{\left|w^{i} \mu_{i j} \Delta^{\frac{\alpha}{2}} w^{j}\right| \geq \theta\left|\Delta^{\frac{\alpha}{2}} w\right|\right\} .
$$


Then, in view of Proposition B.1, for any $\mu \in \Omega$, we have

$$
\begin{aligned}
& \left\|\mu_{i j} w^{j} \Delta^{\frac{\alpha}{2}} w^{i}\right\|_{p_{\alpha}, B_{\Lambda^{-1} r}}^{p_{\alpha}-1} \\
& \quad \approx\left\|\mu_{i j} w^{j} \Delta^{\frac{\alpha}{2}} w^{i}\right\|_{p_{\alpha}, B_{\Lambda^{-1} r} \cap A_{\mu, \theta}}^{p_{\alpha}-1}+\left\|\mu_{i j} w^{j} \Delta^{\frac{\alpha}{2}} w^{i}\right\|_{p_{\alpha}, B_{\Lambda^{-1} r} \cap A_{\mu, \theta}^{c}}^{p_{\alpha}-1}
\end{aligned}
$$

(by Proposition B.1)

$$
\begin{aligned}
& \prec\left\|\mu_{i j} w^{j} \Delta^{\frac{\alpha}{2}} w^{i}\right\|_{p_{\alpha}, B_{\Lambda^{-1} r} \cap A_{\mu, \theta}}^{p_{\alpha}-1}+\sum_{\tilde{\mu} \in \Omega}\left\|\tilde{\mu}_{i j} w^{j} \Delta^{\frac{\alpha}{2}} w^{i}\right\|_{p_{\alpha}, B_{\Lambda^{-1} r} \cap A_{\tilde{\mu}, \theta}}^{p_{\alpha}-1} \\
& +\left\|w^{i} \Delta^{\frac{\alpha}{2}} w^{i}\right\|_{p_{\alpha}, B_{\Lambda^{-1} r}}^{p_{\alpha}-1}
\end{aligned}
$$

(by (2.11))

$$
\prec C_{\theta} \sum_{\tilde{\mu} \in \Omega}\left\|\left|\Delta^{\frac{\alpha}{2}} w\right|^{p_{\alpha}-2} \tilde{\mu}_{i j} w^{j} \Delta^{\frac{\alpha}{2}} w^{i}\right\|_{p_{\alpha^{\prime}}, B_{\Lambda^{-1} 1_{r}}}+\left\|w^{i} \Delta^{\frac{\alpha}{2}} w^{i}\right\|_{p_{\alpha}, B_{\Lambda^{-1} 1_{r}}}^{p_{\alpha}-1} .
$$

Applying again Proposition B.1, we arrive for any $p_{\alpha}>1$ at

$$
\begin{gathered}
\left\|\Delta^{\frac{\alpha}{2}} w\right\|_{p_{\alpha}, B_{\Lambda^{-1} r}}^{p_{\alpha}-1} \prec \sum_{\mu \in \Omega}\left\|\left|\Delta^{\frac{\alpha}{2}} w\right|^{p_{\alpha}-2} \mu_{i j} w^{j} \Delta^{\frac{\alpha}{2}} w^{i}\right\|_{p_{\alpha^{\prime}, B_{\Lambda^{-1} r}}} \\
+\left\|w^{i} \Delta^{\frac{\alpha}{2}} w^{i}\right\|_{p_{\alpha}, B_{\Lambda^{-1} r}}^{p_{\alpha}-1}
\end{gathered}
$$

We conclude by Lemma 2.8 and Lemma 2.2.

The proof of Theorem 2.1 follows now by an iteration argument and an application of Dirichlet's growth theorem (cf. [5], [4, (43)] and [18, arguments after Lemma 6.4]).

\section{A Lower order arguments: Proof of Theorem 1.2}

Let $\alpha \in(0, n)$. In this section, we treat estimates on the bilinear operator

$$
H_{\alpha}(u, v):=\Delta^{\frac{\alpha}{2}}(u v)-u \Delta^{\frac{\alpha}{2}} v-v \Delta^{\frac{\alpha}{2}} u,
$$

which behaves, in some sense, like a product of lower operators (take, for example, the classic case $\alpha=2$ ). The estimates are similar to the ones obtained and used in $[3-5,16,18]$, and here, we will adopt the general strategy of the latter article [18]. The argument relies on the estimates on singular kernels of Proposition A.1. These kernels (the geometric-space analogue to the Fourier-multiplier estimates in [16]) appear because of the following representation of $\Delta^{\frac{\alpha}{2}}, \Delta^{-\frac{\alpha}{2}}$ : For $f \in C_{0}^{\infty}\left(\mathbb{R}^{n}\right)$,

$$
\Delta^{\frac{\alpha}{2}} f(x)=c_{\alpha, n} \int_{\mathbb{R}^{n}} \frac{f(x)-f(y)}{|x-y|^{n+\alpha}} d x \quad \text { if } \alpha \in(0,1),
$$


for some $c_{\alpha, n} \in \mathbb{R} \backslash\{0\}$. The inverse operator of $\Delta^{\frac{\alpha}{2}}$ is the so-called Riesz potential,

$$
\Delta^{-\frac{\alpha}{2}} f(x)=c_{\alpha, n} \int_{\mathbb{R}^{n}} f(\eta)|x-\eta|^{-n+\alpha} d \eta \quad \text { if } \alpha \in(0, n) .
$$

The essential argument appears in the case $\alpha \in(0,1)$. As mentioned above, they are similar to the ones in [18], though there, for convenience, only special cases for $\alpha$ were considered.

\section{A.1 The case $\alpha \in(0,1)$}

With the representation for $\Delta^{\frac{\alpha}{2}}$, one has for $\alpha \in(0,1)$,

$$
\begin{aligned}
\Delta^{\frac{\alpha}{2}}(u v)(x)= & c_{\alpha, n} \int \frac{u(x) v(x)-u(y) v(y)}{|x-y|^{n+\alpha}} d y \\
= & c_{\alpha, n} \int \frac{(u(x)-u(y)) v(x)+u(y)(v(x)-v(y))}{|x-y|^{n+\alpha}} d y \\
= & \Delta^{\frac{\alpha}{2}} u(x) v(x)+c_{\alpha, n} \int \frac{(u(y)-u(x))(v(x)-v(y))}{|x-y|^{n+\alpha}} d y \\
& \quad+\Delta^{\frac{\alpha}{2}} v(x) u(x),
\end{aligned}
$$

that is,

$$
H_{\alpha}(u, v)=c_{\alpha, n} \int \frac{(u(y)-u(x))(v(x)-v(y))}{|x-y|^{n+\alpha}} d y .
$$

Replacing now

$$
u:=\Delta^{-\frac{\alpha}{2}} a, \quad v:=\Delta^{-\frac{\alpha}{2}} b,
$$

this is equivalent to

$$
\begin{aligned}
H_{\alpha}(u, v)=\tilde{c}_{\alpha, n} \iiint & \frac{1}{|x-y|^{n+\alpha}}\left(|y-\eta|^{-n+\alpha}-|x-\eta|^{-n+\alpha}\right) \\
& \times\left(|y-\xi|^{-n+\alpha}-|x-\xi|^{-n+\alpha}\right) a(\eta) b(\xi) d \xi d \eta d y .
\end{aligned}
$$

Thus, the main point of our argument is to replace the differences of functions in the definition of $H$ by differences on the kernels of the respective operators. In the representation above of $H_{\alpha}(u, v)$ it is useful to observe: whenever $|x-y|^{-n-\alpha}$ becomes singular, both the difference of terms with $\eta$ and the terms with $\xi$ tend to zero as well, thus "absorbing" the singularity up to a certain point. More precisely, these kernels can be estimated as in the following proposition. 
Proposition A.1 (Multiplier estimate). Let $\alpha \in[0,1]$ and $\varepsilon \in(0,1)$. Then for almost every $x, y, \eta, \xi \in \mathbb{R}^{n}$, and a uniform constant $C$

$$
\begin{aligned}
& || \eta-\left.y\right|^{-n+\alpha}-|\eta-x|^{-n+\alpha}|||\xi-y|^{-n+\alpha}-|\xi-x|^{-n+\alpha} \mid \\
& \leq C|y-\eta|^{-n+\alpha-\varepsilon}\left(|x-\xi|^{-n+\alpha-\varepsilon}+|y-\xi|^{-n+\alpha-\varepsilon}\right)|x-y|^{2 \varepsilon} \\
& \quad+C\left(|x-\eta|^{-n+\alpha-\varepsilon}+|y-\eta|^{-n+\alpha-\varepsilon}\right)|y-\xi|^{-n+\alpha-\varepsilon}|x-y|^{2 \varepsilon} \\
& \quad+C|x-\eta|^{-n+\alpha}|x-\xi|^{-n+\alpha} \chi_{|x-y|>2|x-\xi| \chi|x-y|>2|x-\eta|}
\end{aligned}
$$

In particular, multiplying this estimate with the hypersingular (i.e. not locally integrable) kernel $|x-y|^{-n-\alpha}$ (the kernel of the differentiation $\Delta^{\frac{\alpha}{2}}$ ), choosing $\varepsilon$ such that $1>2 \varepsilon>\alpha>\varepsilon>0$, the first two terms on the right-hand side consist of products of locally integrable kernels, or, more precisely, kernels of the Riesz potentials $\Delta^{-\frac{\alpha-\varepsilon}{2}}$ and $\Delta^{-\frac{2 \varepsilon-\alpha}{2}}$. In the last term, we then have twice kernels of $\Delta^{-\frac{\alpha}{2}}$, and the kernel $|x-y|^{-n-\alpha} \chi|x-y|>2|x-\xi| \chi|x-y|>2|x-\eta|$ where the singularity $x=y$ is somewhat cut away.

As we will see in Proposition A.2, this enables us, to show that the operators $\Delta^{\frac{\alpha}{2}}$ in the definition of $H_{\alpha}(\cdot, \cdot)$ "distribute their differentiation" on both entryfunctions.

Proof of Proposition A.1. Observe that in the following argument, since $\alpha \in[0,1]$, all the constants can be taken independently from $\alpha$. We set

$$
k(x, y, \eta):=|| \eta-\left.y\right|^{-n+\alpha}-|\eta-x|^{-n+\alpha} \mid .
$$

Decompose the space $(x, y, \eta) \in \mathbb{R}^{3 n}$ into several subspaces depending on the relations of $|y-\eta|,|x-y|,|x-\eta|$ :

$$
1 \leq \chi_{1}(x, y, \eta)+\chi_{2}(x, y, \eta)+\chi_{3}(x, y, \eta) \text { for } x, y, \eta \in \mathbb{R}^{n},
$$

where

$$
\begin{aligned}
& \chi_{1}:=\chi|x-y| \leq 2|y-\eta| \chi|x-y| \leq 2|x-\eta|, \\
& \chi_{2}:=\chi|x-y| \leq 2|y-\eta| \chi|x-y|>2|x-\eta|, \\
& \chi_{3}:=\chi|x-y|>2|y-\eta| \chi|x-y| \leq 2|x-\eta|,
\end{aligned}
$$

and functions of the form $\chi_{f(x, y, \eta)<0}$ denote the usual characteristic functions of the set $\left\{(x, y, \eta) \in \mathbb{R}^{3 N}: f(x, y, \eta)<0\right\}$. Note that with a uniform constant

$$
|y-\eta| \chi_{1} \approx|x-\eta| \chi_{1} .
$$


Then, by the mean value theorem, for the details in this context $\mathrm{cf}$. [18, Proposition 3.3], for any $\varepsilon \in(0,1)$

$$
\begin{aligned}
k(x, y, \eta) \chi_{1} & \prec|x-\eta|^{-n+\alpha-1}|x-y| \chi_{1} \\
& \prec|x-\eta|^{-n+\alpha-\varepsilon}|x-y|^{\varepsilon} \chi_{1} \stackrel{(\mathrm{A} .2)}{\approx}|y-\eta|^{-n+\alpha-\varepsilon}|x-y|^{\varepsilon} \chi_{1} .
\end{aligned}
$$

Moreover, for any $\varepsilon>0$,

$$
k(x, y, \eta) \chi_{2} \prec|x-\eta|^{-n+\alpha} \chi_{2} \prec|x-\eta|^{-n+\alpha-\varepsilon}|x-y|^{\varepsilon},
$$

and also for any $\varepsilon>0$,

$$
k(x, y, \eta) \chi_{3} \prec|y-\eta|^{-n+\alpha} \chi_{3} \prec|y-\eta|^{-n+\alpha-\varepsilon}|x-y|^{\varepsilon} .
$$

In order to estimate now the product $k(x, y, \xi) k(x, y, \eta)$ we have to check the claim for all cases $(i, j), i, j \in\{1,2,3\}$, where we say

$$
\text { case }(i, j) \Leftrightarrow(x, y, \eta, \xi) \in \mathbb{R}^{4 n} \text { such that } \chi_{i}(x, y, \eta) \chi_{j}(x, y, \xi)=1 \text {. }
$$

Let us denote

$$
\begin{aligned}
\text { Type I-estimate } & :=|y-\eta|^{-n+\alpha-\varepsilon}|x-\xi|^{-n+\alpha-\varepsilon}|x-y|^{2 \varepsilon}, \\
\text { Type II-estimate } & :=|y-\eta|^{-n+\alpha-\varepsilon}|y-\xi|^{-n+\alpha-\varepsilon}|x-y|^{2 \varepsilon}, \\
\text { Type III-estimate } & :=|x-\eta|^{-n+\alpha-\varepsilon}|y-\xi|^{-n+\alpha-\varepsilon}|x-y|^{2 \varepsilon}, \\
\text { Type IV-estimate } & :=|x-\eta|^{-n+\alpha}|x-\xi|^{-n+\alpha} \chi|x-y|>2|x-\xi| \chi|x-y|>2|x-\eta| .
\end{aligned}
$$

One checks, that each of these types have to appear. Note, the only case where we choose the Type IV-estimate is $(2,2)$.

As a consequence of Proposition A.1 above, we obtain the following estimate for $\alpha \in(0,1)$ :

Proposition A.2. Let $u=\Delta^{-\frac{\alpha}{2}} \Delta^{\frac{\alpha}{2}} u, v=\Delta^{-\frac{\alpha}{2}} \Delta^{\frac{\alpha}{2}} v$. Then, for $\alpha \in(0,1)$ there exist constants $C_{\alpha}>0, L \equiv L_{\alpha} \in \mathbb{N}, s_{k} \in(0, \alpha), t_{k} \in\left[0, s_{k}\right]$, for $k \in\{1, \ldots, L\}$, such that

$$
\left|H_{\alpha}(u, v)(x)\right| \leq C \sum_{k=1}^{L} \Delta^{-\frac{s_{k}-t_{k}}{2}}\left(\Delta^{-\frac{t_{k}}{2}}\left|\Delta^{\frac{\alpha}{2}} u\right| \Delta^{-\frac{\alpha}{2}+\frac{s_{k}}{2}}\left|\Delta^{\frac{\alpha}{2}} v\right|\right) .
$$

Moreover, $\left|s_{k}-t_{k}\right|$ can be supposed to be arbitrarily small. 
Proof. Set $a:=\Delta^{\frac{\alpha}{2}} u, b:=\Delta^{\frac{\alpha}{2}} v$. By the definition of $\Delta^{\frac{\alpha}{2}}$ for $\alpha \in(0,1)$,

$$
\begin{gathered}
\Delta^{\frac{\alpha}{2}}\left(\Delta^{-\frac{\alpha}{2}} a \Delta^{-\frac{\alpha}{2}} b\right)(x) \\
=c_{n, \alpha} \int \frac{\Delta^{-\frac{\alpha}{2}} a(x) \Delta^{-\frac{\alpha}{2}} b(x)-\Delta^{-\frac{\alpha}{2}} a(y) \Delta^{-\frac{\alpha}{2}} a(y)}{|x-y|^{n+\alpha}} d y \\
=c_{n, \alpha} \int \frac{1}{|x-y|^{n+\alpha}}\left(\left(\Delta^{-\frac{\alpha}{2}} a(x)-\Delta^{-\frac{\alpha}{2}} a(y)\right) \Delta^{-\frac{\alpha}{2}} b(x)\right. \\
\left.\quad+\Delta^{-\frac{\alpha}{2}} a(y)\left(\Delta^{-\frac{\alpha}{2}} b(x)-\Delta^{-\frac{\alpha}{2}} b(y)\right)\right) d y \\
=a(x) \Delta^{-\frac{\alpha}{2}} b(x)+c_{n, \alpha} \int \frac{1}{|x-y|^{n+\alpha}}\left(\Delta^{-\frac{\alpha}{2}} a(y)-\Delta^{-\frac{\alpha}{2}} a(x)\right) \\
\quad \times\left(\Delta^{-\frac{\alpha}{2}} b(x)-\Delta^{-\frac{\alpha}{2}} b(y)\right) d y \\
+b(x) \Delta^{-\frac{\alpha}{2}} a(x) .
\end{gathered}
$$

Consequently,

$$
\begin{aligned}
& \quad\left|H\left(\Delta^{-\frac{\alpha}{2}} a, \Delta^{-\frac{\alpha}{2}} b\right)(x)\right| \\
& \qquad \int_{\mathbb{R}^{n}} \int_{\mathbb{R}^{n}} \int_{\mathbb{R}^{n}} \frac{1}{|x-y|^{n+\alpha}}|| y-\left.\eta\right|^{-n+\alpha}-|x-\eta|^{-n+\alpha} \mid \\
& \quad \times|| y-\left.\xi\right|^{-n+\alpha}-|x-\xi|^{-n+\alpha}|| a|(\eta)| b \mid(\xi) d y d \xi d \eta .
\end{aligned}
$$

Pick $\varepsilon \in(0,1)$ in Proposition A.1 such that

$$
\alpha<2 \varepsilon<\min \{2 \alpha, n+\alpha\} .
$$

Let us see, for instance, how our expression behaves if the "Type I"-estimate from the proof of Proposition A.1 is applicable, that is, if

$$
\begin{gathered}
\frac{|| y-\left.\eta\right|^{-n+\alpha}-|x-\eta|^{-n+\alpha}|||y-\xi|^{-n+\alpha}-|x-\xi|^{-n+\alpha} \mid}{|x-y|^{n+\alpha}} \\
\prec|y-\eta|^{-n+\alpha-\varepsilon}|x-\xi|^{-n+\alpha-\varepsilon}|x-y|^{-n+2 \varepsilon-\alpha} .
\end{gathered}
$$

Observe, by the choice of $\varepsilon$, all the appearing kernels on the right-hand side of this estimate have the exponent $n-\sigma$ for some $\sigma>0$. That is, all the appearing kernels correspond to the kernel of a Riesz potential $\Delta^{-\frac{\sigma}{2}}$, see (A.1). Namely,

$$
\begin{aligned}
& \int|y-\eta|^{-n+\alpha-\varepsilon}|a|(\eta) d \eta \approx \Delta^{-\frac{\alpha-\varepsilon}{2}}|a|(y), \\
& \int|x-\xi|^{-n+\alpha-\varepsilon}|b|(\xi) d \xi \approx \Delta^{-\frac{\alpha-\varepsilon}{2}}|b|(x),
\end{aligned}
$$


and finally

$$
\int|x-y|^{-n+2 \varepsilon-\alpha} \Delta^{-\frac{\alpha-\varepsilon}{2}}|a|(y) d y \approx \Delta^{-\frac{2 \varepsilon-\alpha}{2}} \Delta^{-\frac{\alpha-\varepsilon}{2}}|a|(x) \approx \Delta^{-\frac{\varepsilon}{2}}|a|(x) .
$$

By this kind of strategy, one obtains

$$
\begin{gathered}
\left|H\left(\Delta^{-\frac{\alpha}{2}} a, \Delta^{-\frac{\alpha}{2}} b\right)(x)\right| \prec \Delta^{-\frac{\varepsilon}{2}}|a|(x) \Delta^{-\frac{\alpha-\varepsilon}{2}}|b|(x)+\Delta^{-\frac{\alpha-\varepsilon}{2}}|a|(x) \Delta^{-\frac{\varepsilon}{2}}|b|(x) \\
+\Delta^{-\frac{2 \varepsilon-\alpha}{2}}\left(\Delta^{-\frac{\alpha-\varepsilon}{2}}|a| \Delta^{-\frac{\alpha-\varepsilon}{2}}|b|\right)(x)+A,
\end{gathered}
$$

where

$$
\begin{aligned}
& A:=\int_{\mathbb{R}^{n}} \int_{\mathbb{R}^{n}} \int_{\mathbb{R}^{n}} \frac{|x-\eta|^{-n+\alpha}|x-\xi|^{-n+\alpha}}{|x-y|^{n+\alpha}} \chi|x-y|>2|x-\xi| \\
& \times \chi|x-y|>2|x-\eta||a|(\eta)|b|(\xi) d y d \xi d \eta \\
& \prec \int_{\mathbb{R}^{n}} \int_{\mathbb{R}^{n}}|x-\eta|^{-n+\alpha}|x-\xi|^{-n+\alpha}|x-\xi|^{-\frac{\alpha}{2}}|x-\eta|^{-\frac{\alpha}{2}}|a|(\eta)|b|(\xi) d \xi d \eta \\
& =\Delta^{-\frac{\alpha}{4}}|a|(x) \Delta^{-\frac{\alpha}{4}}|b|(x) \text {. }
\end{aligned}
$$

Thus we can conclude the proof: we choose $L_{\alpha}=3$, with $s_{1}=\varepsilon, t_{1}=\alpha-\varepsilon$, $s_{1}-t_{1}=2 \varepsilon-\alpha$ as first term, then with interchanged roles $s_{2}=t_{1}$ and $t_{2}=s_{2}$, and finally $s_{3}=\alpha / 2, t_{3}=\alpha / 2$, and $s_{2}-t_{2}=2 \varepsilon-\alpha$.

Remark A.3. About the strategy of the proof above let us remark how the decomposition strategy we apply in [18] and here is related to the arguments in [5] and [16]: For the para-product estimates of the bi-commutator in [5], they used an infinite Taylor expansion after Fourier transform in the phase space whenever the symbols were rather close to each other.

Instead, in [16] the mean value formula (that is, a one-step Taylor expansion) was employed, also after Fourier transform, which lead to a simple pointwise estimate in the phase space which sufficed for the purposes there, but did not have the full power of the more complicated, yet more generalizable technique in [5]. Both of these arguments, essentially obtained pointwise bounds in the phase space, which - transformed in the geometric space - gives not pointwise, but $L^{p}\left(\mathbb{R}^{n}\right)$-estimates.

With the method introduced in [18] and employed here, we obtain pointwise results in the geometric space.

But let us stress, that both the arguments in [16] in the phase space and in [18] in the geometric space, which apply both a one-step Taylor expansion, are rough in the following sense: when considering Hardy-space or BMO-space estimates, 
they do not seem to give the optimal result (which is not pointwise anymore), and in this case, the strategy of using para-products as developed in [5] seems more viable, see, e.g., the Hardy-space estimates in [5].

\section{A.2 The case $\alpha \geq 1$}

We will reduce the case $\alpha \geq 1$ to the case $\alpha \in(0,1)$ already discussed. For the case $\alpha=1+\tilde{\alpha} \in[1,2)$, we use the following argument: let $\mathcal{R}_{i}$ be the $i$-th Riesztransform, defined by

$$
\mathcal{R}_{i} f(x)=c \int \frac{(x-y)^{i}}{|x-y|^{n+1}} f(y) d y .
$$

Then for $1+\tilde{\alpha} \in[1,2)$,

$$
\begin{gathered}
\mathcal{R}_{i} H_{1+\tilde{\alpha}}(u, v)=\Delta^{\frac{\alpha}{2}} \partial_{i}(u v)-\mathcal{R}_{i}\left(u \Delta^{\frac{1+\tilde{\alpha}}{2}} v\right)-\mathcal{R}_{i}\left(v \Delta^{\frac{1+\tilde{\alpha}}{2}} u\right) \\
=\Delta^{\frac{\alpha}{2}}\left(u \partial_{i} v\right)-u \Delta^{\frac{\tilde{\alpha}}{2}} \partial_{i} v-\Delta^{\frac{\tilde{\alpha}}{2}} u \partial_{i} v \\
\quad+\Delta^{\frac{\alpha}{2}}\left(v \partial_{i} u\right)-v \Delta^{\frac{\tilde{\alpha}}{2}} \partial_{i} u-\Delta^{\frac{\tilde{\alpha}}{2}} v \partial_{i} u \\
-\mathcal{R}_{i}\left(u \Delta^{\frac{1+\tilde{\alpha}}{2}} v\right)+u \Delta^{\frac{\tilde{\alpha}}{2}} \partial_{i} v \\
-\mathcal{R}_{i}\left(v \Delta^{\frac{1+\tilde{\alpha}}{2}} u\right)+v \Delta^{\frac{\tilde{\alpha}}{2}} \partial_{i} u \\
\quad+\Delta^{\frac{\tilde{\alpha}}{2}} v \partial_{i} u+\Delta^{\frac{\tilde{\alpha}}{2}} u \partial_{i} v \\
=H_{\tilde{\alpha}}\left(u, \partial_{i} v\right)+H_{\tilde{\alpha}}\left(v, \partial_{i} u\right) \\
\quad-\mathcal{R}_{i}\left(u \Delta^{\frac{1+\tilde{\alpha}}{2}} v\right)+u \mathcal{R}_{i} \Delta^{\frac{1+\tilde{\alpha}}{2}} v \\
-\mathcal{R}_{i}\left(v \Delta^{\frac{1+\tilde{\alpha}}{2}} u\right)+v \mathcal{R}_{i} \Delta^{\frac{1+\tilde{\alpha}}{2}} u \\
+\Delta^{\frac{\tilde{\alpha}}{2}} v \partial_{i} u+\Delta^{\frac{\tilde{\alpha}}{2}} u \partial_{i} v .
\end{gathered}
$$

In the case $\tilde{\alpha}=0$, we write this as

$$
\mathcal{R}_{i} H_{1}(u, v)=-\mathcal{R}_{i}\left(u \Delta^{\frac{1}{2}} v\right)+u \mathcal{R}_{i} \Delta^{\frac{1}{2}} \partial_{i} v-\mathcal{R}_{i}\left(v \Delta^{\frac{1}{2}} u\right)+v \mathcal{R}_{i} \Delta^{\frac{1}{2}} u .
$$

The terms $H_{\tilde{\alpha}}(\cdot, \cdot)$, as we've seen above, and the terms $\Delta^{\frac{\tilde{\alpha}}{2}} u \partial_{i} v$ and $\Delta^{\frac{\tilde{\alpha}}{2}} v \partial_{i} u$ are actually products of lower order operators applied to $u$ and $v$, respectively. Moreover, we have the following estimate, which should be compared to the famous commutator estimates and their relation to Hardy spaces and BMO, by Coifman, Rochberg and Weiss [2] and the related work by [1]. 
Proposition A.4. For $\tilde{\alpha} \in[0,1)$ there exist a positive constant $C_{\tilde{\alpha}}$ and a number $L \equiv L_{\tilde{\alpha}} \in \mathbb{N}$, and for $k \in\{1, \ldots, L\}$ constants $s_{k} \in(0,1), t_{k} \in\left[0, s_{k}\right]$ such that

$$
\begin{aligned}
\mathcal{R}_{i}\left(G \Delta^{-\frac{1+\tilde{\alpha}}{2}} F\right)-\left(\mathcal{R}_{i} G \Delta^{-\frac{1+\tilde{\alpha}}{2}} F\right) & \\
& \prec C \sum_{k=1}^{L} \Delta^{-\frac{s_{k}-t_{k}}{2}}\left(\Delta^{-\frac{t_{k}}{2}}|G| \Delta^{-\frac{1+\tilde{\alpha}}{2}+\frac{s_{k}}{2}}|F|\right) .
\end{aligned}
$$

Moreover, $\left|s_{k}-t_{k}\right|$ can be supposed to be arbitrarily small.

Proof. As in the case of $H_{\alpha}$ for $\alpha \in(0,1)$ above, we exploit that the difference of the involved operators can be expressed by the differences of their kernels, more precisely

$$
\begin{aligned}
& \mathcal{R}_{i}\left(G \Delta^{-\frac{1+\tilde{\alpha}}{2}} F\right)(x)-\left(\mathcal{R}_{i} G \Delta^{-\frac{1+\tilde{\alpha}}{2}} F(x)\right. \\
& \quad c \iint \frac{(x-y)^{i}}{|x-y|^{n+1}}\left(|y-z|^{-n+1+\tilde{\alpha}}-|x-z|^{-n+1+\tilde{\alpha}}\right) G(y) F(z) d y d z \\
& \quad \prec c \iint \frac{|| y-\left.z\right|^{-n+1+\tilde{\alpha}}-|x-z|^{-n+1+\tilde{\alpha}} \mid}{|x-y|^{n}}|G|(y)|F|(z) d y d z .
\end{aligned}
$$

Using the mean value theorem, similar to the proof of Proposition A.1, precisely as in [18], we conclude.

In particular, we have

Proposition A.5. Let $u=\Delta^{-\frac{\alpha}{2}} \Delta^{\frac{\alpha}{2}} u, v=\Delta^{-\frac{\alpha}{2}} \Delta^{\frac{\alpha}{2}} v$. Then for $\alpha \in(0,2)$ there exists some constant $C_{\alpha}>0$ and a number $L \equiv L_{\alpha} \in \mathbb{N}$, and for $k \in\{1, \ldots, L\}$ constants $s_{k} \in(0, \alpha), t_{k} \in\left[0, s_{k}\right]$ such that for any $i=1, \ldots, n$, where $\mathcal{R}_{i}$ denotes the Riesz transform,

$$
\left|\mathcal{R}_{i} H_{\alpha}(u, v)(x)\right| \leq C \sum_{k=1}^{L} \Delta^{-\frac{s_{k}-t_{k}}{2}}\left(M_{k} \Delta^{-\frac{t_{k}}{2}} \Delta^{\frac{\alpha}{2}} u N_{k} \Delta^{-\frac{\alpha}{2}+\frac{s_{k}}{2}}\left|\Delta^{\frac{\alpha}{2}} v\right|\right) .
$$

Here, $M_{k}, N_{k}$ are possibly Riesz transforms, or the identity. Moreover, $\left|s_{k}-t_{k}\right|$ can be supposed to be arbitrarily small.

For $\alpha=K+\tilde{\alpha}, \tilde{\alpha} \in(0,2), K \in \mathbb{N}$, observe that

$$
\begin{gathered}
H_{\alpha}(u, v)=\Delta^{\frac{\tilde{\alpha}}{2}}\left(\Delta^{K} u v\right)+\Delta^{\frac{\tilde{\alpha}}{2}}\left(u \Delta^{K} v\right)+\sum_{\substack{|\gamma|+|\tilde{\gamma}|=2 K \\
|\gamma|,|\tilde{\gamma}| \geq 1}} c_{\gamma, \tilde{\gamma}} \Delta^{\frac{\tilde{\alpha}}{2}}\left(\partial^{\gamma} u \partial^{\tilde{\gamma}} v\right) \\
-u \Delta^{K} \Delta^{\frac{\tilde{\alpha}}{2}} v-u \Delta^{K} \Delta^{\frac{\tilde{\alpha}}{2}} v
\end{gathered}
$$




$$
\begin{aligned}
=H_{\tilde{\alpha}}\left(\Delta^{K} u, v\right)+\Delta^{K} u \Delta^{\frac{\tilde{\alpha}}{2}} v+H_{\tilde{\alpha}}\left(u, \Delta^{K} v\right)+\Delta^{K} v \Delta^{\frac{\tilde{\alpha}}{2}} u \\
+\sum_{\substack{|\gamma|+|\tilde{\gamma}|=2 K \\
|\gamma|,|\tilde{\gamma}| \geq 1}} c_{\gamma, \tilde{\gamma}} H_{\tilde{\alpha}}\left(\partial^{\gamma} u, \partial^{\tilde{\gamma}} v\right) \\
+\sum_{\substack{|\gamma|+|\tilde{\gamma}|=2 K \\
|\gamma|,|\tilde{\gamma}| \geq 1}} c_{\gamma, \tilde{\gamma}} \Delta^{\frac{\tilde{\alpha}}{2}} \partial^{\gamma} u \partial^{\tilde{\gamma}} v+\partial^{\gamma} u \Delta^{\frac{\tilde{\alpha}}{2}} \partial^{\tilde{\gamma}} v .
\end{aligned}
$$

Using that all terms which are not of the form $H_{\alpha}$ are actually products of lower order operators, one concludes

Lemma A.6. Let $u=\Delta^{-\frac{\alpha}{2}} \Delta^{\frac{\alpha}{2}} u, v=\Delta^{-\frac{\alpha}{2}} \Delta^{\frac{\alpha}{2}} v$. Then for $\alpha \in(0, n)$ there exist some constant $C_{\alpha}>0$ and a number $L \equiv L_{\alpha} \in \mathbb{N}$, and for $k \in\{1, \ldots, L\}$ constants $s_{k} \in(0, \alpha), t_{k} \in\left[0, s_{k}\right]$ such that for any $i=1, \ldots, n$,

$$
\left|\mathcal{R}_{i} H_{\alpha}(u, v)(x)\right| \leq C \sum_{k=1}^{L} M_{k} \Delta^{-\frac{s_{k}-t_{k}}{2}}\left(\Delta^{-\frac{t_{k}}{2}}\left|\Delta^{\frac{\alpha}{2}} u\right| N_{k} \Delta^{-\frac{\alpha}{2}+\frac{s_{k}}{2}}\left|\Delta^{\frac{\alpha}{2}} v\right|\right) .
$$

Here, $M_{k}, N_{k}$ are possibly Riesz transforms, or the identity. Moreover, $\left|s_{k}-t_{k}\right|$ can be supposed to be arbitrarily small.

In particular:

Proposition A.7. Let $\alpha \in(0, n), q, q_{1}, q_{2} \in[1, \infty]$ such that

$$
\frac{1}{q}=\frac{1}{q_{1}}+\frac{1}{q_{2}} \text {. }
$$

Then

$$
\left\|H_{\alpha}(u, v)\right\|_{\left(\frac{n}{\alpha}, q\right), \mathbb{R}^{n}} \prec\left\|\Delta^{\frac{\alpha}{2}} u\right\|_{\left(\frac{n}{\alpha}, q_{2}\right), \mathbb{R}^{n}}\left\|\Delta^{\frac{\alpha}{2}} v\right\|_{\left(\frac{n}{\alpha}, q_{2}\right), \mathbb{R}^{n}} .
$$

\section{A.3 Local estimates}

In this section, the goal is to give in Lemma A.9 a localized version of Proposition A.7.

Similar to the arguments in Proposition 2.6, one can show

Proposition A.8. There is $\gamma>0$ such that for any $\Lambda>4, s \in(0, \alpha), t \in[0, s]$,

$$
\left\|\Delta^{-\frac{s-t}{2}}\left(\Delta^{-\frac{t}{2}}\left|\eta_{-\Lambda r} a\right| \Delta^{-\frac{\alpha}{2}+\frac{s}{2}}|b|\right)\right\|_{\frac{n}{\alpha}, B_{r}} \prec \Lambda^{-\gamma}\|a\|_{\frac{n}{\alpha}}\|b\|_{\frac{n}{\alpha}}
$$

and for $\Lambda_{1}, \Lambda_{2}>4$

$$
\left\|\Delta^{-\frac{s-t}{2}}\left(\Delta^{-\frac{t}{2}}\left|\eta_{-\Lambda_{1} r} a\right| \Delta^{-\frac{\alpha}{2}+\frac{s}{2}}\left(\eta_{-\Lambda_{2} r}|b|\right)\right)\right\|_{\frac{n}{\alpha}, B_{r}} \prec \Lambda_{1}^{-\gamma} \Lambda_{2}^{-\gamma}\|a\|_{\frac{n}{\alpha}}\|b\|_{\frac{n}{\alpha}} .
$$


Proof. For some $\psi \in C_{0}^{\infty}\left(B_{r}\right),\|\psi\|_{\frac{n}{n-\alpha}} \leq 1$ we have to estimate (up to possibly Riesz transforms, which we will ignore again, as they do not change the argument)

$$
\begin{aligned}
& \int \Delta^{-\frac{s-t}{2}}\left(\Delta^{-\frac{t}{2}}\left|\eta_{-\Lambda} a\right| \Delta^{-\frac{\alpha}{2}+\frac{s}{2}}|b|\right) \psi \\
& =\int \Delta^{-\frac{t}{2}}\left|\eta_{-\Lambda} a\right| \Delta^{-\frac{\alpha}{2}+\frac{s}{2}}|b| \Delta^{-\frac{s-t}{2}} \psi \\
& \prec\left\|\eta_{\sqrt{\Lambda}} \Delta^{-\frac{t}{2}}\left|\eta_{-\Lambda} a\right|\right\|_{\frac{n}{\alpha-t}}\left\|\Delta^{-\frac{\alpha}{2}+\frac{s}{2}}|b|\right\|_{\frac{n}{s}}\left\|\Delta^{-\frac{s-t}{2}} \psi\right\|_{\frac{n}{n-\alpha-s+t}} \\
& +\left\|\Delta^{-\frac{t}{2}}\left|\eta_{-\Lambda} a\right|\right\|_{\frac{n}{\alpha-t}}\left\|\Delta^{-\frac{\alpha}{2}+\frac{s}{2}}|b|\right\|_{\frac{n}{s}}\left\|\left(1-\eta_{\sqrt{\Lambda}}\right) \Delta^{-\frac{s-t}{2}} \psi\right\|_{\frac{n}{n-\alpha-s+t}} \\
& \prec\left\|\eta_{\sqrt{\Lambda}} \Delta^{-\frac{t}{2}}\left|\eta_{-\Lambda} a\right|\right\|_{\frac{n}{\alpha-t}}\|b\|_{\frac{n}{\alpha}}+\|a\|_{\frac{n}{\alpha}}\|b\|_{\frac{n}{\alpha}}\left\|\left(1-\eta_{\sqrt{\Lambda}}\right) \Delta^{-\frac{s-t}{2}} \psi\right\|_{\frac{n}{n-\alpha-s+t}}
\end{aligned}
$$

Then the first claim follows the arguments for products of (non-local) fractional operators with disjoint support, cf., e.g., the arguments of Proposition 2.3 or [18, Proposition 4.4].

The second claim follows by the same method, only taking more care in the cutoff for $b$.

Lemma A.9. Let $v, w \in W^{\alpha, p}\left(\mathbb{R}^{n}\right)$. Then for any $\Lambda>2$

$$
\begin{aligned}
&\|H(v, w)\|_{\frac{n}{\alpha}, B_{r}} \prec\left\|\eta_{\Lambda r} \Delta^{\frac{\alpha}{2}} v\right\|_{\frac{n}{\alpha}}\left\|\eta_{\Lambda r} \Delta^{\frac{\alpha}{2}} w\right\|_{\frac{n}{\alpha}} \\
&+\Lambda^{-\gamma}\left\|\Delta^{\frac{\alpha}{2}} w\right\|_{\frac{n}{\alpha}}\left\|\eta_{\Lambda r} \Delta^{\frac{\alpha}{2}} v\right\|_{\frac{n}{\alpha}} \\
&+\Lambda^{-\gamma}\left\|\Delta^{\frac{\alpha}{2}} v\right\|_{\frac{n}{\alpha}}\left\|\eta_{\Lambda r} \Delta^{\frac{\alpha}{2}} w\right\|_{\frac{n}{\alpha}} \\
&+\Lambda^{-\gamma}\left\|\Delta^{\frac{\alpha}{2}} v\right\|_{\frac{n}{\alpha}} \sum_{k=1}^{\infty} 2^{-k \gamma}\left\|\eta_{\Lambda r}^{k} \Delta^{\frac{\alpha}{2}} w\right\|_{\frac{n}{\alpha}} .
\end{aligned}
$$

Proof. Set

$$
\begin{aligned}
v_{\Lambda} & :=\Delta^{-\frac{\alpha}{2}}\left(\eta_{\Lambda r} \Delta^{\frac{\alpha}{2}} v\right), \\
v_{-\Lambda} & :=\Delta^{-\frac{\alpha}{2}}\left(\left(1-\eta_{\Lambda r}\right) \Delta^{\frac{\alpha}{2}} v\right), \\
v_{-\Lambda, k} & :=\Delta^{-\frac{\alpha}{2}}\left(\eta_{\Lambda r}^{k} \Delta^{\frac{\alpha}{2}} v\right) .
\end{aligned}
$$

Then

$$
H(v, w)=H\left(v_{\Lambda}, w_{\Lambda}\right)+H\left(v_{-\Lambda}, w_{\Lambda}\right)+H\left(v_{\Lambda}, w_{-\Lambda}\right)+H\left(v_{-\Lambda}, w_{-\Lambda}\right)
$$

We have

$$
\left\|H\left(v_{\Lambda}, w_{\Lambda}\right)\right\|_{\frac{n}{\alpha}} \prec\left\|\eta_{\Lambda r} \Delta^{\frac{\alpha}{2}} v\right\|_{\frac{n}{\alpha}}\left\|\eta_{\Lambda r} \Delta^{\frac{\alpha}{2}} w\right\|_{\frac{n}{\alpha}}
$$


Moreover, the second and the third term are controlled by means of Proposition A.8. It remains to estimate $H\left(v_{-\Lambda}, w_{-\Lambda}\right)$,

$$
H\left(v_{-\Lambda}, w_{-\Lambda}\right)=\sum_{k=1}^{\infty} H\left(v_{-\Lambda}, w_{-\Lambda, k}\right)
$$

and again by Proposition A.8,

$$
\left\|H\left(v_{-\Lambda}, w_{-\Lambda, k}\right)\right\|_{\frac{n}{\alpha}, B_{r}} \prec 2^{-\gamma k} \Lambda^{-2 \gamma}\|v\|_{\frac{n}{\alpha}}\left\|\eta_{\Lambda r}^{k} \Delta^{\frac{\alpha}{2}} w\right\|_{\frac{n}{\alpha}} .
$$

\section{B Decomposition in Euclidean spaces}

In the proof of Lemma 2.9 we used the following fact, which permitted us to get a full information of $\left\|\Delta^{\frac{\alpha}{2}} w\right\|_{p_{\alpha}}$ from the information of $\left\|\mu_{i j} w^{j} \Delta^{\frac{\alpha}{2}} w^{i}\right\|_{p_{\alpha}}$, and the normal projection $\left\|w^{i} \Delta^{\frac{\alpha}{2}} w^{i}\right\|_{p_{\alpha}}$.

Proposition B.1. Let $\vec{p} \in \mathbb{R}^{N},|\vec{p}|=1$. Set

$$
\Omega:=\left\{\mu \equiv\left(\mu_{i j}\right)_{i, j=1}^{N}: \mu_{i j}=-\mu_{i j} \in\{-1,0,1\}\right\},
$$

and let for $\mu \in \Omega$

$$
\vec{p}_{\mu} \equiv\left(\vec{p}_{\mu_{i}}\right)_{i=1}^{N}:=\left(\mu_{i j} \vec{p}^{j}\right)_{i=1}^{N} .
$$

Then, for uniform constants $c, C$, depending only on the dimension $N$,

$$
c|\vec{q}| \leq \sum_{\mu \in \Omega}\left|\left\langle\vec{p}_{\mu}, \vec{q}\right\rangle\right|+|\langle\vec{p}, \vec{q}\rangle| \leq C|\vec{q}| \quad \text { for all } \vec{q} \in \mathbb{R}^{N} .
$$

In particular, there is a uniform $\theta \in(0,1)$ such that for any $\vec{q} \in \mathbb{R}^{N}$, if for some $\mu \in \Omega$,

$$
\left|\left\langle\vec{p}_{\mu}, \vec{q}\right\rangle\right| \leq \theta|\vec{q}|,
$$

then there exists $\tilde{\mu} \in \Omega$, such that

$$
\left|\left\langle\vec{p}_{\mu}, \vec{q}\right\rangle\right| \leq \theta|\vec{q}| \leq \max \left\{\left|\left\langle\vec{p}_{\tilde{\mu}}, \vec{q}\right\rangle\right|,|\langle\vec{p}, \vec{q}\rangle|\right\} .
$$

For the convenience of the reader, we will give a proof.

Proof. We prove the first inequality of the claim (B.1) for $|\vec{q}|=1$ and $\vec{q} \perp \vec{p}$. The case $N=1$ is trivial, of course, so assume $N \geq 2$. Let for $\alpha \neq \beta$,

$$
\mu_{i, j}^{\alpha \beta}:=\delta_{i}^{\alpha} \delta_{j}^{\beta}-\delta_{j}^{\alpha} \delta_{i}^{\beta} \in \Omega,
$$


where $\delta$ denotes the Kronecker symbol

$$
\delta_{\alpha}^{i}= \begin{cases}1 & \text { if } \alpha=i \\ 0 & \text { else. }\end{cases}
$$

The claim (B.1) then follows if we can show that there exists a uniform constant $c>0$ such that vor any $\vec{p} \equiv\left(\vec{p}_{i}\right)_{i=1}^{N}, \vec{q} \equiv\left(\vec{q}_{i}\right)_{i=1}^{N} \in \mathbb{R}^{N}$ with $|\vec{p}|=|\vec{q}|=1$, and $\vec{p} \perp \vec{q} \in \mathbb{R}^{N}$, there are $\alpha \neq \beta$ such that

$$
\left|\left\langle\vec{p}_{\mu^{\alpha \beta}}, \vec{q}\right\rangle_{\mathbb{R}^{N}}\right| \equiv\left|\vec{p}_{\alpha} \vec{q}_{\beta}-\vec{p}_{\beta} \vec{q}_{\alpha}\right| \geq c .
$$

Choose $i_{0} \neq k_{0}$ such that (w.l.o.g) $\vec{p}_{i_{0}}, \vec{q}_{k_{0}}>0$ and, more important,

$$
\vec{p}_{i_{0}}, \vec{q}_{k_{0}} \geq \frac{1}{2 \sqrt{N-1}}=: c_{0} .
$$

In fact, by orthonormality of $\vec{p} \perp \vec{q}$, it cannot happen that for some $i$, both

$$
\left|\vec{p}_{i}\right|^{2},\left|\vec{q}_{i}\right|^{2} \geq \frac{3}{4} \text {. }
$$

Thus, if there is some $i_{0}$ such that $\left|\vec{p}_{i_{0}}\right|^{2} \geq \frac{3}{4}$, then $\vec{q}_{i_{0}}^{2} \leq \frac{3}{4}$ and because of $|\vec{q}|=1$ there has to exist at least one $k_{0} \neq i_{0}$ such that $\vec{q}_{k_{0}}$ satisfies (B.2). An analogous argument holds if $\left|\vec{q}_{k_{0}}\right|^{2} \geq \frac{3}{4}$ for some $k_{0}$. In the remaining cases, we can assume that $\left|\vec{p}_{i}\right|^{2},\left|\vec{q}_{k}\right|^{2}<\frac{3}{4}$ for all $i, k$. But then there have to be at least two indices $i_{0} \neq i_{1}$ such that $\vec{p}_{i}$ satisfies (B.2) for $i=i_{1}, i_{1}$ : because if this was not the case, we had the following contradiction:

$$
1=\sum_{i=1}^{N}\left|\vec{p}_{i}\right|^{2}<\frac{3}{4}+(N-1) \frac{1}{4(N-1)}=1 .
$$

We thus have two different choices for both for $i_{0}$ in order for $\vec{p}_{i_{0}}$ to satisfy (B.2), so whichever the choice of $k_{0}$ is, we can choose $i_{0} \neq k_{0}$. Then,

$$
\left\langle\vec{p}_{\mu^{i} k^{k_{0}}}, \vec{q}\right\rangle_{\mathbb{R}^{N}}=\vec{p}_{i_{0}} \vec{q}_{k_{0}}-\vec{p}_{k_{0}} \vec{q}_{i_{0}} .
$$

If $\vec{p}_{k_{0}} \vec{q}_{i_{0}} \leq 0$, this implies

$$
\left\langle\vec{p}_{\mu^{i} k_{0} k_{0}}, \vec{q}\right\rangle_{\mathbb{R}^{N}} \geq\left(c_{0}\right)^{2} .
$$

Assume on the other hand that $\vec{p}_{k_{0}} \vec{q}_{i_{0}}>0$ and that even

$$
\left\langle\vec{p}_{\mu^{i_{0} k_{0}}}, \vec{q}\right\rangle \leq \frac{1}{10}\left(c_{0}\right)^{2} \text {. }
$$

Then,

$$
\left|\vec{p}_{k_{0}} \vec{q}_{i_{0}}\right|=\vec{p}_{k_{0}} \vec{q}_{i_{0}}=\vec{p}_{i_{0}} \vec{q}_{k_{0}}-\left\langle\vec{p}_{\mu^{i_{0} k_{0}}}, \vec{q}\right\rangle_{\mathbb{R}^{N}} \geq\left(c_{0}\right)^{2}-\frac{1}{10}\left(c_{0}\right)^{2} .
$$


Since $\left|\vec{p}_{k_{0}}\right|,\left|\vec{q}_{i_{0}}\right| \leq 1$, we infer

$$
\left|\vec{p}_{k_{0}}\right|,\left|\vec{q}_{i_{0}}\right| \geq \frac{9}{10}\left(c_{0}\right)^{2}
$$

It follows that

$$
c_{1}:=\frac{9}{10}\left(c_{0}\right)^{3} \leq\left|\vec{q}_{i_{0}}\right|\left|\vec{p}_{i_{0}}\right|+\left|\vec{q}_{k_{0}}\right|\left|\vec{p}_{k_{0}}\right| .
$$

Because of orthogonality $\vec{p} \perp \vec{q}$,

$$
-\left(\vec{q}_{i_{0}} \vec{p}_{i_{0}}+\vec{q}_{k_{0}} \vec{p}_{k_{0}}\right)=\sum_{i \neq i_{0}, k_{0}} \vec{p}_{i} \vec{q}_{i}
$$

As the product $\vec{p}_{k_{0}} \vec{q}_{i_{0}}>0$, we have to consider the case $I$ where both $\vec{p}_{k_{0}}, \vec{q}_{i_{0}}>0$ and the case II where both $\vec{p}_{k_{0}}, \vec{q}_{i_{0}}<0$. Recall that in both cases $\vec{p}_{i_{0}}, \vec{q}_{k_{0}}>0$. As for case $I$, (B.4), (B.3) imply

$$
\begin{aligned}
-c_{1} & \geq \sum_{\substack{i \neq i_{0}, k_{0} \\
\vec{p}_{i} \vec{q}_{i}<0}} \vec{p}_{i} \vec{q}_{i}+\sum_{\substack{i \neq i_{0}, k_{0} \\
\vec{p}_{i} \vec{q}_{i}>0}} \vec{p}_{i} \vec{q}_{i}=-\sum_{\substack{i \neq i_{0}, k_{0} \\
\vec{p}_{i} \vec{q}_{i}<0}}\left|\vec{p}_{i}\right|\left|\vec{q}_{i}\right|+\sum_{\substack{i \neq i_{0}, k_{0} \\
\vec{p}_{i} \vec{q}_{i}>0}}\left|\vec{p}_{i}\right|\left|\vec{q}_{i}\right| \\
& \geq-\sum_{\substack{i \neq i_{0}, k_{0} \\
\vec{p}_{i} \vec{q}_{i}<0}}\left|\vec{p}_{i}\right|\left|\vec{q}_{i}\right| .
\end{aligned}
$$

In particular, this implies that there is $i_{1} \neq i_{0}, k_{0}$ such that $\vec{p}_{i_{1}} \vec{q}_{i_{1}}<0$ and still (recall $\left|\vec{p}_{i_{1}}\right|,\left|\vec{q}_{i_{1}}\right| \leq 1$ )

$$
\left|\vec{p}_{i_{1}}\right|,\left|\vec{q}_{i_{1}}\right| \geq \frac{c_{1}}{N} .
$$

Assuming $\vec{p}_{i_{1}}>0, \vec{q}_{i_{1}}<0$, since we know $\vec{q}_{i_{0}}>0, \vec{p}_{i_{0}}>0$, we have

$$
\left\langle\vec{p}_{\mu^{i_{1} i_{0}}}, \vec{q}\right\rangle_{\mathbb{R}^{N}}=\vec{p}_{i_{1}} \vec{q}_{i_{0}}-\vec{p}_{i_{0}} \vec{q}_{i_{1}}>\left|\vec{p}_{i_{0}}\right|\left|\vec{q}_{i_{1}}\right| \geq c_{0} \frac{c_{1}}{N} .
$$

If we assume, on the other hand, $\vec{p}_{i_{1}}>0, \vec{q}_{i_{1}}<0$,

$$
-\left\langle\vec{p}_{\mu^{i_{1} i_{0}}}, \vec{q}\right\rangle_{\mathbb{R}^{N}}=-\vec{p}_{i_{1}} \vec{q}_{i_{0}}+\vec{p}_{i_{0}} \vec{q}_{i_{1}}<-\left|\vec{p}_{i_{0}}\right|\left|\vec{q}_{i_{1}}\right| \leq-c_{0} \frac{c_{1}}{N} .
$$

In case $I I$, where both $\vec{p}_{k_{0}}, \vec{q}_{i_{0}}<0$, we have instead

$$
c_{1} \leq-\sum_{\substack{i \neq i_{0}, k_{0} \\ \vec{p}_{i} \vec{q}_{i}<0}}\left|\vec{p}_{i}\right|\left|\vec{q}_{i}\right|+\sum_{\substack{i \neq i_{0}, k_{0} \\ \vec{p}_{i} \vec{q}_{i}>0}}\left|\vec{p}_{i}\right|\left|\vec{q}_{i}\right| \leq \sum_{\substack{i \neq i_{0}, k_{0} \\ \vec{p}_{i} \vec{q}_{i}>0}}\left|\vec{p}_{i}\right|\left|\vec{q}_{i}\right|,
$$


and we can find $i_{2} \neq i_{0}, k_{0}$ such that $\vec{p}_{i_{2}} \vec{q}_{i_{2}}>0$ and

$$
\left|\vec{p}_{i_{2}}\right|,\left|\vec{q}_{i_{2}}\right| \geq \frac{c_{1}}{N}
$$

Then,

$$
-\operatorname{sign}\left(\vec{p}_{i_{2}}\right)\left\langle\vec{p}_{\mu^{i_{2} i_{0}}}, \vec{q}\right\rangle_{\mathbb{R}^{N}}=\left|\vec{p}_{i_{2}}\right|\left|\vec{q}_{i_{0}}\right|+\vec{p}_{i_{0}}\left|\vec{q}_{i_{2}}\right| \geq c_{0} \frac{c_{1}}{N}
$$

This proves the first claim, which essentially is contained in the following: for $|\vec{p}|=1$, the finite set $\left\{\vec{p}_{\mu}: \mu \in \Omega\right\}$ may not be a basis; nevertheless, it is a linear generator of the space $\vec{p}^{\perp} \subset \mathbb{R}^{N}$.

For the second claim we use the following argument: since $\Omega$ is finite, any vector $\vec{q}$ of length $|\vec{q}|=1$ has to have length at least $\theta=\frac{1}{|\Omega|+1}$ in at least one of the linear spaces generated by some $\vec{p}_{\mu}$ or generated by $\vec{p}$ itself. That is $\left|\left\langle\vec{p}_{\mu}, \vec{q}\right\rangle\right| \geq \theta$ for some $\mu \in \Omega$, or $|\langle\vec{p}, \vec{q}\rangle| \geq \theta$. Consequently, if there exists some $\mu$ such that $\left|\left\langle\vec{p}_{\mu}, \vec{q}\right\rangle\right|<\theta$, then there has to be another $\tilde{\mu} \in \Omega$ such that $\left|\left\langle\vec{p}_{\tilde{\mu}}, \vec{q}\right\rangle\right| \geq \theta$ or, alternatively, $|\langle\vec{p}, \vec{q}\rangle| \geq \theta$.

\section{Bibliography}

[1] S. Chanillo, A note on commutators, Indiana Univ. Math. J. 31 (1982), 7-16.

[2] R. R. Coifman, R. Rochberg and G. Weiss, Factorization theorems for Hardy spaces in several variables, Ann. of Math. (2) 103 (1976), 611-635.

[3] F. Da Lio, Fractional harmonic maps into manifolds in odd dimension $n>1$, Calc. Var. Partial Differential Equations, 2012, DOI: 10.1007/s00526-012-0556-6.

[4] F. Da Lio and T. Rivière, Sub-criticality of non-local Schrödinger systems with antisymmetric potentials and applications to half-harmonic maps, Adv. Math. 227 (2011), 1300-1348.

[5] F. Da Lio and T. Rivière, Three-term commutator estimates and the regularity of 1/2-harmonic maps into spheres, Anal. PDE 4 (2011), 149-190.

[6] F. Da Lio and A. Schikorra, (n,p)-harmonic maps into manifolds. The case $p<2$, in preparation.

[7] E. Di Nezza, G. Palatucci and E. Valdinoci, Hitchhiker's guide to the fractional Sobolev spaces, Bull. Sci. Math. 136 (2012), 521-573.

[8] F. Duzaar and G. Mingione, Local Lipschitz regularity for degenerate elliptic systems, Ann. Inst. H. Poincaré Anal. Non Linéaire 27 (2010), 1361-1396.

[9] J. Frehse, A discontinuous solution of a mildly nonlinear elliptic system, Math. Z. 134 (1973), 229-230.

[10] F. Hélein, Régularité des applications faiblement harmoniques entre une surface et une sphère, C. R. Acad. Sci. Paris Sér. I 311 (1990), 519-524. 
[11] S. Hofmann, An off-diagonal $T 1$ theorem and applications, J. Funct. Anal. 160 (1998), 581-622.

[12] T. Kato and G. Ponce, Commutator estimates and the Euler and Navier-Stokes equations, Comm. Pure Appl. Math. 41 (1988), 891-907.

[13] S. Kolasiński, Regularity of weak solutions of $n$-dimensional $H$-systems, Differential Integral Equations 23 (2010), no. 11-12, 1073-1090.

[14] T. Rivière, Conservation laws for conformally invariant variational problems, Invent. Math. 168 (2007), 1-22.

[15] T. Rivière, The role of integrability by compensation in conformal geometric analysis, in: Analytic Aspects of Problems from Riemannian Geometry S.M.F., to appear.

[16] A. Schikorra, Regularity of $n / 2$-harmonic maps into spheres, J. Differential Equations 252 (2012), 1862-1911.

[17] A. Schikorra, A note on regularity for the $n$-dimensional $H$-system assuming logarithmic higher integrability, preprint (2010), http: //arxiv . org/abs/1012 . 2737.

[18] A. Schikorra, Interior and boundary-regularity for fractional harmonic maps on domains, preprint (2011), http://arxiv.org/abs/1103.5203.

[19] P. Strzelecki, Regularity of $p$-harmonic maps from the $p$-dimensional ball into a sphere, Manuscripta Math. 82 (1994), 407-415.

[20] P. Strzelecki, On biharmonic maps and their generalizations, Calc. Var. Partial Differential Equations 18 (2003), 401-432.

[21] C. Wang, A compactness theorem of $n$-harmonic maps, Ann. Inst. H. Poincaré Anal. Non Linéaire 22 (2005), 509-519.

Received February 16, 2012; revised August 7, 2012; accepted September 1, 2012.

\section{Author information}

Francesca Da Lio, Department of Mathematics, ETH Zürich,

Rämistr. 101, 8092 Zürich, Switzerland;

and Dipartimento di Matematica Pura ed Applicata, Università degli Studi di Padova,

Via Trieste 63, 35121, Padova, Italy.

E-mail: fdalio@math.ethz.ch

Armin Schikorra, Max-Planck Institut MIS Leipzig,

Inselstr. 22, 04315 Leipzig, Germany.

E-mail: armin.schikorra@mis.mpg.de 